\title{
SNOD: A Fast Sampling Method of Exploring Node Orbit Degrees for Large Graphs
}

\author{
Pinghui Wang · Junzhou Zhao · Xiangliang \\ Zhang • Jing Tao • Xiaohong Guan
}

Received: date / Accepted: date

\begin{abstract}
Exploring small connected and induced subgraph patterns (CIS patterns, or graphlets) has recently attracted considerable attention. Despite recent efforts on computing how frequent a graphlet appears in a large graph (i.e., the total number of CISes isomorphic to the graphlet), little effort has been made to characterize a node's graphlet orbit degree, i.e., the number of CISes isomorphic to the graphlet that touch the node at a particular orbit, which is an important fine-grained metric for analyzing complex networks such as learning functions/roles of nodes in social and biological networks. Like global graphlet counting, it is computationally intensive to compute node orbit degrees for a large graph. Furthermore, previous methods of computing global graphlet counts are not suited to solve this problem. In this paper, we propose a novel sampling method SNOD to efficiently estimate node orbit degrees for large-scale graphs, and quantify the error of our estimates. To the best of our knowledge, we are the first to study this problem and give a fast scalable solution. We conduct experiments on a variety of real-world datasets and demonstrate that our method SNOD is several orders of magnitude faster than state-of-the-art enumeration methods for accurately estimating node orbit degrees for graphs with millions of edges.
\end{abstract}

Keywords Graph mining · sampling · graphlet

Pinghui Wang, Jing Tao, Xiaohong Guan

NSKEYLAB, Xi' an Jiaotong University, Xi'an, Shaanxi, China

Shenzhen Research School, Xi'an Jiaotong University, Shenzhen, Guangdong, China

Tel.: +086-2982664603

Fax: +086-2982664603

E-mail: \{phwang, jtao, xhguan\}@mail.xjtu.edu.cn

Junzhou Zhao, Xiangliang Zhang

King Abdullah University of Science and Technology, Thuwal, SA

E-mail: junzhouzhao@gmail.com,xiangliang.zhang@kaust.edu.sa

Junzhou Zhao and Jing Tao are the corresponding authors. 


\section{Introduction}

Exploring patterns in a graph is important for understanding and exploring networks such as online social networks (OSNs) and computer networks. For example, the presence of significant homophily (i.e., the tendency of users to connect to others with common interests) has been discovered in a variety of real-world networks, which indicates that nodes in a network could be organized based on underlying communities they belong to. Recently, $[6,15,28]$ reveal that nodes could also be organized based on structural roles of nodes in the network. For example, in Figure 1, nodes $u, v$, and $w$ are hubs in three communities learned by minimizing the number of cutting edges (i.e., the edges with two endpoints within different communities). We observe $u$ is more structurally similar to $w$ than $v$, and $x$ and $y$ exhibit similar structure roles. Grover and Leskovec [14] observe that leveraging on structural role information can significantly improve the performance of machine learning tasks such as node classification and link prediction.

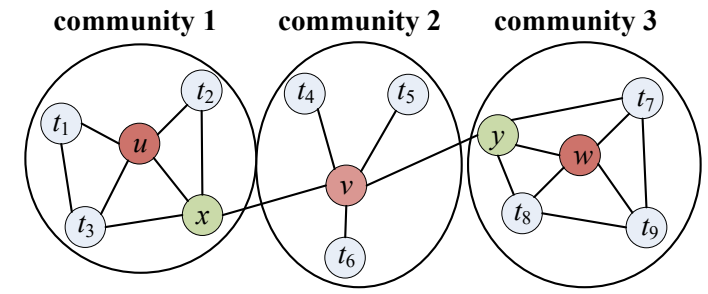

orbit degrees

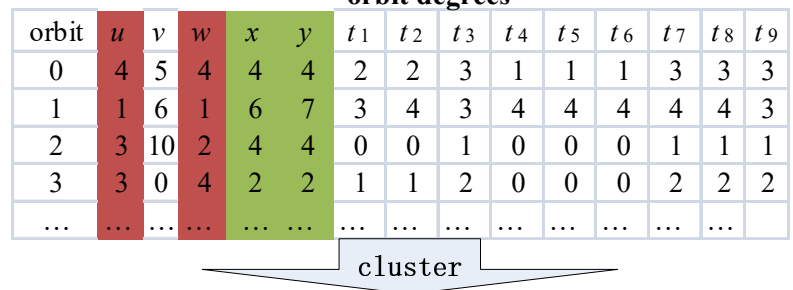

role cluster 1: $\{u, w\}$; role cluster 2: $\{v, x, y\}$; role cluster 3: $\left\{t_{1}, \cdots, t_{9}\right\}$

Fig. 1 Example of structure roles. $u, v$, and $w$ are hub nodes in three communities learned by minimizing the number of cutting edges, while node orbit degrees reflect that $u$ is more structurally similar to $w$ than $v$, and $x$ and $y$ exhibit similar structure roles.

Exploring connected subgraph patterns (i.e., motifs, also known as graphlets) is an effective way to study structure roles of nodes in a network [6,28]. As shown in Figure 2, there is one 2-node graphlet $G_{0}$, two 3-node graphlets $G_{1}$ and $G_{2}$, and six 4-node graphlets $G_{3}, \ldots, G_{8}$, and these graphlets are widely used for characterizing networks' local connection patterns. However, nodes may occupy very different positions in the same graphlet. For example, the three leaf nodes (in black) of $G_{4}$ in Figure 2 are symmetric, therefore their positions belong to the same class. The other node (in white) of $G_{4}$ behaves more like a hub. According to the positions that nodes of a graphlet occupy, Przulj et al. [28] group the graphlet's nodes into one or more 


\begin{tabular}{|c|c|c|cccccc|}
\hline 2-node & \multicolumn{3}{|c|}{3 -node } & \multicolumn{5}{c|}{ 4-node } \\
$G_{0}$ & $G_{1}$ & $G_{2}$ & $G_{3}$ & $G_{4}$ & $G_{5}$ & $G_{6}$ & $G_{7}$ & $G_{8}$ \\
\hline
\end{tabular}

Fig. 2 Graphlets and their automorphism orbits studied in this paper. Numbers in blue are orbit IDs. There is one 2-node graphlet $G_{0}$, two 3-node graphlets $G_{1}$ and $G_{2}$, and six 4-node graphlets $G_{3}, \ldots, G_{8}$. Nodes may occupy very different positions in the same graphlet. For example, the three leaf nodes (in black) of $G_{4}$ are symmetric. and the other node (in white) of $G_{4}$ exhibits more like a hub. According to the positions that nodes of a graphlet occupy, the graphlet's nodes are classified into one or more different orbits (i.e., position classes) associated with them. The values of orbit IDs have no specific meaning, and we set the same values of orbit IDs as [28].

different automorphism orbits $^{1}$ (i.e., position classes). They observe that a node's orbit degree vector, or orbit degree signature, which counts the number of connected and induced subgraphs (CISes) that touch the node at a particular orbit, is an important metric for representing the node's topology features. For example, we compute all nodes' orbit degrees in Figure 1 and observe that the orbit degrees reflect that $u$ and $x$ are most structurally similar to $w$ and $y$ respectively. In addition, clustering nodes according to their orbit degrees also provides us valuable insights for deeply understanding the network. For instance, the role cluster $\{u, w\}$ learned in Figure 1 probably consists of nodes who are hubs in closely connected communities, and the role cluster $\{x, y, v\}$ probably consists of nodes who exhibit like structure holes, i.e., nodes act as mediators between two or more connected communities of nodes. In fact, the orbit degree signature has been successfully used for protein function prediction [23] and cancer gene identification [22] by identifying groups (or clusters) of topologically similar nodes in biological networks. In addition to biological networks, orbit degree is also used for link prediction [41] and node classification [12] in OSNs, as well as hyponym relation extraction from Wikipedia hyperlinks [39].

However, it is computationally intensive to enumerate and compute orbit degrees for large graphs, because nodes may be included in a large number of CISes. For example, there exist at least $d_{v}^{2} / 2$ 3-node CISes and $d_{v}^{3} / 34$-node CISes that include a particular node $v$ having $d_{v}$ neighbors, therefore it is computationally expensive to compute orbit degrees of nodes with a large number of neighbors. To solve this challenge, other methods such as sampling could be used in place of the brute-force enumeration approach. Despite recent progress in counting specific graphlets such as triangles $[35,5,16,2]$ and 4-node graphlets [18] that appear in a large graph, little attention has been given to developing fast tools for computing orbit degrees. Existing methods of estimating global graphlet counts cannot be used for computing this finegrained metric, because: 1) it is not easy to derive and correct their sampling biases for estimating each node's orbit degrees; 2) they are not able to set a fine-grained sampling ratio for each node in the large graph of interest, which leads to nodes included by a large/small number of CISes over/under-sampled. Moreover, sometimes one may only want to explore orbit degrees of a particular node (e.g., Lady Gaga

1 The values of orbit IDs in Figure 2 have no specific meaning. We set the values of orbit IDs same as [28]. 
on Twitter). Existing methods are customized to sample all CISes in a large graph, but not tailored to solving this problem. The straightforward solution is to apply these sampling methods to the neighborhood subgraph of the node $v$ of interest, where the neighborhood subgraph of $v$ consists of nodes that can reach $v$ within $k$ (the maximum size of graphlets of interest) steps, and the edges between these nodes in the original graph. Unfortunately, we observe that the neighborhood subgraph still contains a large fraction of CISes not including $v$, which leads to waste a lot of time sampling these irrelevance CISes for existing methods.

To solve the above problem, inspired by sampling based graphlet counting methods $[17,18,38]$, we design a scalable and computationally efficient method, SNOD, to estimate node orbit degrees for large graphs. The basic idea behind these sampling based graphlet counting methods $[17,18,38]$ can be briefly summarized as: Suppose that a sampling method is able to randomly sample a CIS isomorphic to a specific graphlet $i$ with the same probability $p_{i}$ from all CISes in graph $G=(V, E)$ of interest. One can obtain $k$ random CISes by running the sampling method $k$ times independently, where $k$ is the sampling budget specified in advance. Let $m_{i}$ be the number of sampled CISes isomorphic to graphlet $i$. Then, $\frac{m_{i}}{k p_{i}}$ is an unbiased estimate of the count of graphlet $i$, i.e., the number of CISes isomorphic to graphlet $i$. Therefore, the graphlet counting problem boils down to how to design a fast sampling method of which $p_{i}$ is simple and easy to be derived and computed. For example, wedge sampling [17] is designed for approximately counting triangles. It first uniformly and randomly samples a path consisting of 2 edges and then retrieves the CIS consisting of the 3 nodes on the path. Any triangle in $G$ is sampled with probability $p=\frac{3}{W}$, where $W=\sum_{v \in V} \frac{d_{v}\left(d_{v}-1\right)}{2}$. Similarly, 3-path sampling [18], designed for counting 4-node CISes, first uniformly and randomly samples a path consisting of 3 edges and then retrieves the CIS consisting of the 4 nodes on the path. Any CIS isomorphic to graphlet $i$ in $G$ is sampled with probability $\frac{\varphi_{i}}{\Gamma}$, where $\Gamma=\sum_{(u, v) \in E}\left(d_{u}-1\right)\left(d_{v}-1\right)$ and $\varphi_{i}$ is the number of different 3 -edge paths in graphlet $i$. Inspired by the above methods, we extend wedge sampling to sample CISes including node $v \in V$ of interest at orbits 1, 2, and 3. Similarly, we develop a method Path3, which extends 3-path sampling to sample CISes including node $v$ at orbits 5 to 14 . However, Path3 cannot sample CISes including node $v$ at all orbits 5 to 14 such as orbits 6 and 7. To solve this problem, we develop another method Star3 to sample CISes including node $v$ at other orbits. Based on CISes sampled by these three sampling methods, we propose a method to estimate degrees of orbits 1 to 14 for node $v$. More importantly, we also derive expressions for variances of our estimates, which is of great value in practice since the variances can be used to bound the estimates' errors and determine the smallest necessary sampling budget for a given desired accuracy. We conduct experiments on a variety of publicly available datasets. Our experimental results show that SNOD is several orders of magnitude faster than state-of-the-art enumeration methods for accurately estimating orbit degrees. We demonstrate the ability of SNOD to explore large graphs with millions of nodes and edges.

The rest of this paper is organized as follows. Section 2 presents the problem formulation. Section 3 introduces preliminaries used in this paper. Section 4 presents our method SNOD for estimating node orbit degrees. Section 5 presents the perfor- 
mance evaluation and testing results. Section 6 summarizes related work. Concluding remarks then follow.

\section{Problem Formulation}

Denote the undirected graph of interest as $G=(V, E)$, where $V$ is a set of nodes and $E \in V \times V$ is a set of edges. In order to define orbit degrees, we first introduce some notation. A subgraph $G^{\prime}$ of $G$ is a graph whose set of nodes and set of edges are both subsets of $G$. An induced subgraph of $G, G^{\prime}=\left(V^{\prime}, E^{\prime}\right)$, is a subgraph that consists of a subset of nodes in $G$ and all those edges that connect them in $G$, i.e. $V^{\prime} \subset V$, $E^{\prime}=\left\{(u, v): u, v \in V^{\prime},(u, v) \in E\right\}$. Unless we explicitly say "induced" in this paper, a subgraph is not necessarily induced. Figure 2 shows all 2-, 3-, and 4-node graphlets $G_{i}, 0 \leq i \leq 8$, in [28]. By taking into account the "symmetries" between nodes in $G_{i}$, Przulj [28] classifies the nodes of $G_{i}$ into different automorphism orbits (or just obits, for brevity), where the nodes with the same orbit ID are topologically identical. For all $G_{i}, 0 \leq i \leq 8$, there are 15 orbits, which are shown in Figure 2 . Denote $C_{v}^{(i)}$ as the set of connected and induced subgraphs (CISes) in $G$ that touch a node $v \in V$ at orbit $i$. Let $d_{v}^{(i)}=\left|C_{v}^{(i)}\right|$ denote the graphlet orbit $i$ degree (or just "orbit $i$ degree", for brevity) of $v$. The orbit degree vector, $\left(d_{v}^{(0)}, \ldots, d_{v}^{(14)}\right)$, can be used as a signature of node $v$ for applications such as identifying similar nodes. We observe that $C_{v}^{(0)}$ contains the edges in $G$ that include node $v$, i.e., $C_{v}^{(0)}=\{(u, v)$ : $(u, v) \in E\}$, and $d_{v}^{(0)}$ is the number of neighbors of $v$. For simplicity, we denote $d_{v}=d_{v}^{(0)}$ as the degree of node $v$. An example is given in Figure 3, where $d_{v}^{(0)}=3$, $d_{v}^{(2)}=2, d_{v}^{(1)}=d_{v}^{(3)}=d_{v}^{(5)}=d_{v}^{(10)}=d_{v}^{(11)}=1$, and $d_{v}^{(4)}=d_{v}^{(6)}=d_{v}^{(7)}=d_{v}^{(8)}=$ $d_{v}^{(9)}=d_{v}^{(12)}=d_{v}^{(13)}=d_{v}^{(14)}=0$.

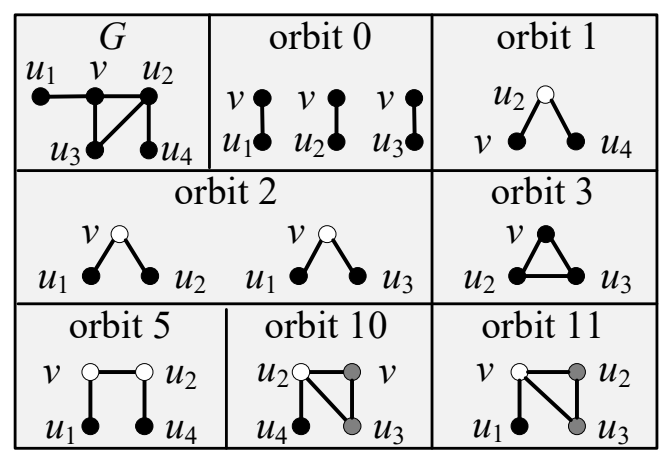

Fig. 3 Example of computing the orbit degrees of node $v$ in graph $G$.

As discussed above, it is computationally intensive to enumerate and count all 3and 4-node CISes that include a given node with a large number of neighbors in large graphs. As we will show, later in our experiments the node with the largest degree in graph Flickr [20] belongs to more than $10^{13}$ 3- and 4-node CISes. In this paper, 
we develop a computationally efficient method SNOD to estimate node orbit degrees for large graphs. For ease of reading, we list notation used throughout the paper in Table 1.

Table 1 Table of notation.

\begin{tabular}{|c|c|}
\hline$G=(V, E)$ & $G$ is the graph of interest \\
\hline$N_{v}$ & the set of neighbors of a node $v$ in $G$ \\
\hline$d_{v}$ & degree of node $v$, i.e., $d_{v}=\left|N_{v}\right|$ \\
\hline$G_{0}, \ldots, G_{8}$ & 2-, 3-, and 4-node graphlets \\
\hline$C_{v}^{(i)}, i=1, \ldots, 14$ & the set of CISes in $G$ that touch a node $v$ at orbit $i$ \\
\hline$d_{v}^{(1)}, \ldots, d_{v}^{(14)}$ & orbit degrees of node $v$ \\
\hline$\pi_{1, v}, \ldots, \pi_{14, v}$ & orbit probability distribution of sampling method Path2 $(v, G)$ \\
\hline$\check{\pi}_{1, v}, \ldots, \check{\pi}_{14, v}$ & orbit probability distribution of sampling method Path3 $(v, G)$ \\
\hline$\tilde{\pi}_{1, v}, \ldots, \tilde{\pi}_{14, v}$ & orbit probability distribution of sampling method $\operatorname{Star} 3(v, G)$ \\
\hline$k, \tilde{k}, \tilde{k}$ & sampling budgets of Path2 $(v, G), \operatorname{Path} 3(v, G), \operatorname{Star} 3(v, G)$ \\
\hline$m_{i}, \check{m}_{i}, \tilde{m}_{i}$ & $\begin{array}{l}\text { the number of CISes sampled by Path2 }(v, G) \text {, Path3 }(v, G) \text {, and } \\
\text { Star3 }(v, G) \text { that include } v \text { in orbit } i \text { respectively }\end{array}$ \\
\hline$v_{\max }$ & the node with the largest degree in $G$ \\
\hline$\phi_{v}$ & the number of CISes consisting of $v$ and any two neighbors of $v$ \\
\hline$\psi_{v}$ & the number of paths of length 3 starting with node $v$ \\
\hline$\gamma_{v}$ & the number of CISes consisting of $v$ and any three neighbors of $v$ \\
\hline$\varphi_{v}$ & the number of paths of length 2 starting with node $v$ \\
\hline$\check{\varphi}_{v}$ & the number of paths of length 3 including $v$ as the second node \\
\hline$\tilde{\varphi}_{v}$ & $\begin{array}{l}\text { the number of 4-node subgraphs that are isomorphic to a 4-node } \\
\text { star (i.e., graphlet } G_{4} \text { ) and include } v \text { as a leaf node }\end{array}$ \\
\hline $\boldsymbol{\alpha}^{(v)}=\left(\alpha_{u}^{(v)}\right)_{u \in N_{v}}$ & sampling distribution used by methods $\operatorname{Path} 2(v, G)$ and $\operatorname{Path} 3(v, G)$ \\
\hline $\boldsymbol{\beta}^{(v)}=\left(\beta_{u}^{(v)}\right)_{u \in N_{v}}$ & sampling distribution used by method $\operatorname{Star} 3(v, G)$ \\
\hline
\end{tabular}

\section{Preliminaries}

In this section, we introduce two theorems that provide the theoretical foundation for our methods in estimating orbit degrees.

Theorem 1 (Estimating subset cardinalities) Let $C^{(1)}, \ldots, C^{(h)}$ be $h$ sets of interest. Let $d^{(i)}=\left|C^{(i)}\right|$ denote the cardinality of $C^{(i)}, 1 \leq i \leq h$. Suppose that there exists no element in common for any two sets $C^{(i)}$ and $C^{(j)}, 1 \leq i<j \leq h$, while there exists a function $\mathbb{F}$ that returns a random element $x$ sampled from $C=$ $C^{(1)} \cup \ldots \cup C^{(h)}$ according to a distribution $P\left(x=s \wedge s \in C^{(i)}\right)=p_{i}$, where $\sum_{i=1}^{h} d^{(i)} p_{i}=1$. Let $x_{1}, \ldots, x_{k}$ be random elements obtained by calling function $\mathbb{F}$ $k$ times independently. Denote by $\mathbf{1}(\mathbb{P})$ the indicator function that equals one when predicate $\mathbb{P}$ is true, and zero otherwise. When $p_{i}>0$, we estimate $n_{i}$ as:

$$
\hat{d}^{(i)}=\frac{\sum_{j=1}^{k} \mathbf{1}\left(x_{j} \in C^{(i)}\right)}{k p_{i}}, \quad 1 \leq i \leq h,
$$


where $\hat{d}^{(i)}$ is an unbiased estimator of $d^{(i)}$ (i.e., $\left.\mathbb{E}\left(\hat{d}^{(i)}\right)=d^{(i)}\right)$ with variance $\operatorname{Var}\left(\hat{d}^{(i)}\right)=\frac{d^{(i)}}{k}\left(\frac{1}{p_{i}}-d^{(i)}\right)$. The covariance of $\hat{d}^{(i)}$ and $\hat{d}^{(j)}$ is $\operatorname{Cov}\left(\hat{d}^{(i)}, \hat{d}^{(j)}\right)=$ $-\frac{d^{(i)} d^{(j)}}{k}, i \neq j, i, j=1, \ldots, h$.

Proof. We easily have

$$
P\left(x_{j} \in C^{(i)}\right)=\sum_{s \in C^{(i)}} P\left(x_{j}=s \wedge s \in C^{(i)}\right)=p_{i} d^{(i)}, \quad 1 \leq i \leq h, 1 \leq j \leq k .
$$

Since $x_{1}, \ldots, x_{k}$ are sampled independently, the random variable $\sum_{j=1}^{k} \mathbf{1}\left(x_{j} \in\right.$ $\left.C^{(i)}\right)$ follows the binomial distribution with parameters $k$ and $p_{i} d^{(i)}$. Then, the expectation and variance of $\sum_{j=1}^{k} \mathbf{1}\left(x_{j} \in C^{(i)}\right)$ are

$$
\begin{gathered}
\mathbb{E}\left(\sum_{j=1}^{k} \mathbf{1}\left(x_{j} \in C^{(i)}\right)\right)=k p_{i} d^{(i)}, \\
\operatorname{Var}\left(\sum_{j=1}^{k} \mathbf{1}\left(x_{j} \in C^{(i)}\right)\right)=k p_{i} d^{(i)}\left(1-p_{i} d^{(i)}\right) .
\end{gathered}
$$

Therefore, the expectation and variance of $\hat{d}^{(i)}$ are computed as

$$
\begin{gathered}
\mathbb{E}\left(\hat{d}^{(i)}\right)=\mathbb{E}\left(\frac{\sum_{j=1}^{k} \mathbf{1}\left(x_{j} \in C^{(i)}\right)}{k p_{i}}\right)=d^{(i)} \\
\operatorname{Var}\left(\hat{d}^{(i)}\right)=\operatorname{Var}\left(\frac{\sum_{j=1}^{k} \mathbf{1}\left(x_{j} \in C^{(i)}\right)}{k p_{i}}\right)=\frac{d^{(i)}}{k}\left(\frac{1}{p_{i}}-d^{(i)}\right) .
\end{gathered}
$$

For any $i \neq j$ and $1 \leq i, j \leq h$, the covariance of $\hat{d}^{(i)}$ and $\hat{d}^{(j)}$ is

$$
\begin{aligned}
\operatorname{Cov}\left(\hat{d}^{(i)}, \hat{d}^{(j)}\right) & =\operatorname{Cov}\left(\frac{\sum_{t=1}^{k} \mathbf{1}\left(x_{t} \in C^{(i)}\right)}{k p_{i}}, \frac{\sum_{l=1}^{k} \mathbf{1}\left(x_{l} \in C^{(j)}\right)}{k p_{j}}\right) \\
& =\frac{\sum_{t=1}^{k} \operatorname{Cov}\left(\mathbf{1}\left(x_{t} \in C^{(i)}\right), \mathbf{1}\left(x_{t} \in C^{(j)}\right)\right)}{k^{2} p_{i} p_{j}} \\
& =-\frac{d^{(i)} d^{(j)}}{k} .
\end{aligned}
$$

In the derivation above, we use

$$
\begin{aligned}
& \operatorname{Cov}\left(\mathbf{1}\left(x_{t} \in C^{(i)}\right), \mathbf{1}\left(x_{l} \in C^{(j)}\right)\right)=0, \quad t \neq l \\
& \operatorname{Cov}\left(\mathbf{1}\left(x_{t} \in C^{(i)}\right), \mathbf{1}\left(x_{t} \in C^{(j)}\right)\right) \\
&= \mathbb{E}\left(\mathbf{1}\left(x_{t} \in C^{(i)}\right) \mathbf{1}\left(x_{t} \in C^{(j)}\right)\right)-\mathbb{E}\left(\mathbf{1}\left(x_{t} \in C^{(i)}\right)\right) \mathbb{E}\left(\mathbf{1}\left(x_{t} \in C^{(j)}\right)\right) \\
&= 0-p_{i} d^{(i)} p_{j} d^{(j)} \\
&=-p_{i} p_{j} d^{(i)} d^{(j)} .
\end{aligned}
$$


Theorem 2 (Combining unbiased estimators [13]) Suppose there exist b independent and unbiased estimates $\mu_{1}, \ldots, \mu_{b}$ of $\mu$ with variances $\operatorname{Var}\left(\mu_{j}\right), j=1, \ldots, b$. Let $\alpha_{i}=\frac{\operatorname{Var}^{-1}\left(\mu_{i}\right)}{\sum_{j=1}^{b} \operatorname{Var}^{-1}\left(\mu_{j}\right)}$. The estimate $\hat{\mu}=\sum_{j=1}^{b} \alpha_{j} \mu_{j}$ is the minimum variance estimate based on a linear combination of $\mu_{1}, \ldots, \mu_{b}$. Its variance is $\operatorname{Var}(\hat{\mu})=$ $\frac{1}{\sum_{j=1}^{k} \operatorname{Var}^{-1}\left(\mu_{j}\right)}$.

\section{Sampling Node Orbits}

The fundamental principle of our solution is: the problem of computing node orbit degrees can be addressed as estimating subset cardinalities. Let $S(v)$ denote the set of all 3- and 4-node CISes that include a given node $v \in V$ of interest. Let $S_{i}(v) \in S(v)$ denote the set of CISes that include $v$ in orbit $i=1, \ldots, 14$ (Figure 2). The orbit $i$ degree of $v$ is exactly the cardinality of $S_{i}(v)$. Theorem 1 suggests that $\left|S_{i}(v)\right|$ has an unbiased estimate when sampling according to a probability distribution $P(x=$ $\left.s \wedge s \in S_{i}(v)\right)$, which can be easily derived and efficiently computed. Then the key of the solution is to design a fast method to sample $S_{i}(v)$.

In this section, we present three fast sampling methods Path2 $(v, G)$, Path3 $(v, G)$, and $\operatorname{Star} 3(v, G)$, which are building blocks of our method SNOD. Path2 $(v, G)$ fast samples a 3-node CIS that includes a path of length 2 starting with node $v$. Clearly, it is able to sample 3-node CISes that include $v$ in orbits 1 and 3. Path3( $v, G)$ fast samples a 4-node CIS that includes a path of length 3 with $v$ as the second node. $\operatorname{Star} 3(v, G)$ fast samples a 4-node CIS that includes a subgraph isomorphic to a 4-node star with $v$ as a leaf node. As shown in Table 2, each of Path3 $(v, G)$ and $\operatorname{Star} 3(v, G)$ is only able to sample 4-node CISes that include $v$ in a subset of orbits. However, together they are able to sample all CISes that include $v$ in orbits $\{4, \ldots, 14\}-\{4,7\}$. Based on samples obtained by methods Path2 $(v, G), \operatorname{Path} 3(v, G)$, and $\operatorname{Star} 3(v, G)$, we easily estimate the degrees of $v$ in orbits $\{1, \ldots, 14\}-\{2,4,7\}$ according to Theorem 1 . To estimate the degrees of $v$ in orbits $\{2,4,7\}$, we rely on three relationships between orbit degrees shown in Theorem 3 .

Table 2 Summary of graphlet orbit sampling methods in this paper.

\begin{tabular}{|c|c|c|c|c|c|c|c|c|c|c|c|c|c|c|}
\hline \multirow{2}{*}{ method } & \multicolumn{14}{|c|}{ whether the method is able to sample a CIS that includes $v$ in orbit $i$} \\
\hline & 1 & 2 & 3 & 4 & 5 & 6 & 7 & 8 & 9 & 10 & 11 & 12 & 13 & 14 \\
\hline $\operatorname{Path} 2(v, G)$ & $\checkmark$ & $\times$ & $\checkmark$ & $\times$ & $\times$ & $\times$ & $\times$ & $\times$ & $\times$ & $\times$ & $\times$ & $\times$ & $\times$ & $\times$ \\
\hline Path3 $(v, G)$ & $\times$ & $\times$ & $\checkmark$ & $\times$ & $\checkmark$ & $\times$ & $\times$ & $\checkmark$ & $\times$ & $\checkmark$ & $\checkmark$ & $\checkmark$ & $\checkmark$ & $\checkmark$ \\
\hline $\operatorname{Star} 3(v, G)$ & $\times$ & $\times$ & $\times$ & $\times$ & $\times$ & $\checkmark$ & $\times$ & $\times$ & $\checkmark$ & $\checkmark$ & $\times$ & $\checkmark$ & $\checkmark$ & $\checkmark$ \\
\hline
\end{tabular}

Theorem 3 For a node $v \in V$ in graph $G$, denote $\phi_{v}$ as the number of CISes consisting of $v$ and any two neighbors of $v, \gamma_{v}$ as the number of CISes consisting of $v$ and any three neighbors of $v$, and $\psi_{v}$ as the number of paths of length 3 starting with node $v$. Formally, $\phi_{v}, \gamma_{v}$, and $\psi_{v}$ are computed as

$$
\phi_{v}=\frac{d_{v}\left(d_{v}-1\right)}{2}
$$




$$
\begin{gathered}
\gamma_{v}=\frac{d_{v}\left(d_{v}-1\right)\left(d_{v}-2\right)}{6}, \\
\psi_{v}=\sum_{u \in N_{v}} \sum_{w \in N_{u}-\{v\}}\left(d_{w}-1\right) .
\end{gathered}
$$

Then, we have

$$
\begin{gathered}
d_{v}^{(2)}+d_{v}^{(3)}=\phi_{v} \\
d_{v}^{(7)}+d_{v}^{(11)}+d_{v}^{(13)}+d_{v}^{(14)}=\gamma_{v} \\
2 d_{v}^{(3)}+d_{v}^{(4)}+2 d_{v}^{(8)}+2 d_{v}^{(9)}+d_{v}^{(10)}+4 d_{v}^{(12)}+2 d_{v}^{(13)}+6 d_{v}^{(14)}=\psi_{v} .
\end{gathered}
$$

Proof. We easily find that a 3-node CIS $s$ consisting of $v$ and any two neighbors of $v$ includes $v$ in orbit 2 or 3 . Therefore, we have $d_{v}^{(2)}+d_{v}^{(3)}=\left(\begin{array}{c}d_{v} \\ 2\end{array}\right)=\phi_{v}$. Similarly, we easily find that a 4-node CIS $s$ consisting of $v$ and any three neighbors of $v$ includes $v$ in orbit 7, 11, 13, or 14. Therefore, we have $d_{v}^{(7)}+d_{v}^{(11)}+d_{v}^{(13)}+d_{v}^{(14)}=\left(\begin{array}{c}d_{v} \\ 3\end{array}\right)=\gamma_{v}$. Let $\Delta$ be the set of paths of length 3 starting with node $v$, i.e., $\Delta=\{(v, u, w, r)$ : $(v, u),(u, w),(w, r) \in E, v \neq w, u \neq r\}$. Then, we easily have $\psi_{v}=|\Delta|=$ $\sum_{u \in N_{v}} \sum_{w \in N_{u}-\{v\}}\left(d_{w}-1\right)$. For CISes including $v$ in orbits $3,4,8,9,10,12,13$, and 14, Figure 4 shows that they include $2,1,2,2,1,4,2$, and 6 paths of length 3 starting with node $v$ respectively. We easily find that the other CISes do not include any path of length 3 starting with node $v$. Therefore, we have Eq. (3).

\begin{tabular}{|l|l|l|l|l|}
\hline$v$ & path $1: u=u_{1}, w=u_{2}, r=v$ \\
$u_{2}$
\end{tabular}

Fig. 4 Paths of length 3 starting with node $v$, (i,e., $(v, u),(u, w),(w, r) \in E, v \neq w$ and $u \neq r$ ) in a CIS that includes $v$ in different orbits. Numbers in blue are orbit IDs.

Formally, our method SNOD consists of the following three steps:

Step 1. Apply function Path $2(v, G) k$ times to sample $k$ CISes;

Step 2. Apply function Path $3(v, G) \check{k}$ times to sample $\check{k}$ CISes;

Step 3. Apply function $\operatorname{Star} 3(v, G) \tilde{k}$ times to sample $\tilde{k}$ CISes;

Step 4. Estimate $d_{v}^{(1)}, \ldots, d_{v}^{(14)}$ based on samples of Path2 $(v, G), \operatorname{Path} 3(v, G)$, and $\operatorname{Star} 3(v, G)$.

Next, we introduce each of the above steps in detail. The time complexity of each of functions $\operatorname{Path} 2(v, G)$, Path3 $(v, G)$, and Path3 $(v, G)$ are summarized in Table 3, which will be discussed in detail in Appendix. The total time complexity of SNOD is $O\left(d_{v}+(k+\check{k}+\tilde{k}) \log d_{v}\right)$. 
Table 3 Time complexities of sampling methods in this paper.

\begin{tabular}{|c|c|c|}
\hline \multirow{2}{*}{ method } & \multicolumn{2}{|c|}{ computational complexity } \\
\cline { 2 - 3 } & initialization & sample one CIS \\
\hline Path2 $(v, G)$ & 0 & $O\left(\log d_{v}\right)$ \\
\hline Path3 $(v, G)$ & $O\left(d_{v}\right)$ & $O\left(\log d_{v}\right)$ \\
\hline Star3 $(v, G)$ & $O\left(d_{v}\right)$ & $O\left(\log d_{v}\right)$ \\
\hline
\end{tabular}

\subsection{Sampling Method Path2 $(v, G)$}

Denote $\varphi_{v}$ as the number of paths of length 2 starting with node $v$. Formally, $\varphi_{v}$ is computed as

$$
\varphi_{v}=\sum_{u \in N_{v}}\left(d_{u}-1\right)
$$

Path2 $(v, G)$ first randomly samples a path of length 2 starting with node $v$, and then retrieves the path's 3-node induced subgraph. Formally, Path2 $(v, G)$ consists of three steps:

Step 1. Sample a node $u$ from $N_{v}$ according to distribution $\boldsymbol{\alpha}^{(v)}=\left(\alpha_{u}^{(v)}\right)_{u \in N_{v}}$, where we define

$$
\alpha_{u}^{(v)}=\frac{d_{u}-1}{\varphi_{v}} .
$$

Here we do not sample $u$ from $N_{v}$ uniformly but according to $\boldsymbol{\alpha}^{(v)}$ to guarantee that the first two steps of Path2 $(v, G)$ randomly and uniformly samples a path of length 2 starting with node $v$, which facilitates estimation of the sampling bias;

Step 2. Sample a node $w$ from $N_{u}-\{v\}$ at random;

Step 3. Return the CIS $s$ consisting of nodes $v, u$, and $w$.

The pseudo-code of our method Path2 $(v, G)$ is shown in Algorithm 1, where function WeightRandomVertex $\left(N_{v}, \boldsymbol{\alpha}^{(v)}\right)$ returns a node sampled from $N_{v}$ according to distribution $\boldsymbol{\alpha}^{(v)}$, and function RandomVertex $\left(N_{u}-\{v\}\right)$ returns a node sampled from $N_{u}-\{v\}$ at random. In Appendix, we introduce the implementation of these functions in detail. The following theorem 4 specifies the sampling probability distribution of Path2 $(v, G)$.

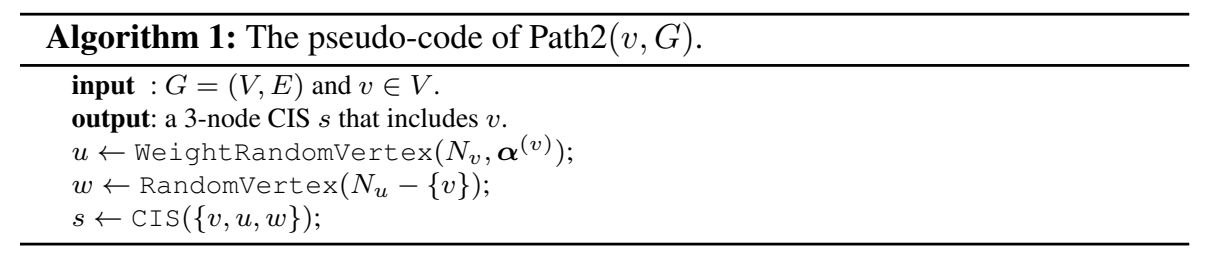

Theorem 4 Let $\pi_{i, v}$ denote the probability that method Path $2(v, G)$ samples a 3 node CIS $s$ including $v$ in orbit $i \in\{1,2,3\}$. Then $\pi_{1, v}=\frac{1}{\varphi_{v}}, \pi_{2, v}=0$, and $\pi_{3, v}=\frac{2}{\varphi_{v}}$. 
Proof. The number of selections of variables $u$ and $w$ in Algorithm 1, i.e., the number of paths of length 2 starting with node $v$, is $\varphi_{v}=\sum_{u \in N_{v}}\left(d_{u}-1\right)$. Let $s$ be a 3-node CIS consisting three nodes $v, u_{1}$, and $u_{2}$. When $s$ includes $v$ in orbit 3 (i.e., $s$ is a triangle), Path2 $(v, G)$ has two different ways to sample $s$ : (1) $u=u_{1}$ and $w=u_{2}$; (2) $u=u_{2}$ and $w=u_{1}$. Each one happens with probability $\frac{1}{\varphi_{v}}$. When $s$ includes $v$ in orbit 1 (i.e., $s$ is a path of length 2 starting with $v)$, $\operatorname{Path} 2(v, G)$ has only one way to sample $s$, which happens with probability $\alpha_{u}^{(v)} \times \frac{1}{d_{u}-1}=\frac{1}{\varphi_{v}}$. When $s$ includes $v$ in orbit 2 (i.e., $s$ is a path of length 2 and $v$ is in the middle of the path), $\operatorname{Path} 2(v, G)$ is not able to sample it. Therefore, we have $\pi_{1, v}=\frac{1}{\varphi_{v}}, \pi_{2, v}=0$, and $\pi_{3, v}=\frac{2}{\varphi_{v}}$.

\begin{tabular}{|c|c|c|}
\hline$\overbrace{u_{1}}^{v} \begin{array}{l}\text { case } 1: u=u_{1}, w=u_{2}, r=u_{2} \\
\text { case } 2: u=u_{2}, w=u_{1}, r=u_{1}\end{array}$ & ${ }_{u_{2}}^{v} \overbrace{u_{3}}^{u_{1}} \quad$ case $1: u=u_{1}, w=u_{2}, r=u_{3}$ & $\bigsqcup_{u_{2} u_{3}}^{v}$ case $1: u=u_{1}, w=u_{2}, r=u_{3}$ \\
\hline$\overbrace{u_{2}}^{u_{1}} v_{u_{3}}^{v}{ }^{10}$ case $1: u=u_{1}, w=u_{3}, r=u_{2}$ & $\begin{array}{lll}v & u_{1} \\
& 10 & \text { case } 1: u=u_{1}, w=u_{2}, r=u_{3} \\
u_{2} & u_{3} & \text { case } 2: u=u_{3}, w=u_{2}, r=u_{1}\end{array}$ & $\begin{array}{ccc}u_{1} & u_{3} & \text { case } 1: u=u_{1}, w=u_{2}, r=u_{3} \\
12 & u_{2} & \text { case } 2: u=u_{2}, w=u_{1}, r=u_{3}\end{array}$ \\
\hline 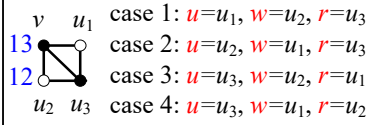 & $\begin{array}{l}\text { case 1: } u=u_{1}, w=u_{2}, r=u_{3} \\
\text { case 2: } u=u_{1}, w=u_{3}, r=u_{2} \\
\text { case 3: } u=u_{2}, w=u_{1}, r=u_{3}\end{array}$ & $\begin{array}{l}\text { case 4: } u=u_{2}, w=u_{3}, r=u_{1} \\
\text { case 5: } u=u_{3}, w=u_{1}, r=u_{2} \\
\text { case 6: } u=u_{3}, w=u_{2}, r=u_{1}\end{array}$ \\
\hline
\end{tabular}

Fig. 5 The ways of $\operatorname{Path} 3(v, G)$ sampling a CIS that includes $v$ in different orbits. Numbers in blue are orbit IDs. $u, w$, and $r$ in red are the variables in Algorithm 2.

\begin{tabular}{|c|c|c|c|}
\hline$\overbrace{v}^{u_{1}} u_{2}$ & $\begin{array}{l}\text { case 1: } u=u_{1}, w=u_{2}, r=u_{3} \\
\text { case 2: } u=u_{1}, w=u_{3}, r=u_{2}\end{array}$ & $\underbrace{u_{1}}_{v} u_{3} u_{2}$ case $2: u=u_{1}, w=u_{3}, r=u_{2}$ & 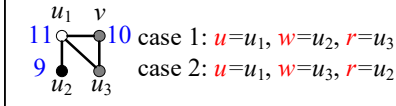 \\
\hline \multirow[t]{2}{*}{ vis } & $\begin{array}{l}\text { case 1: } u=u_{1}, w=u_{2}, r=u_{3} \\
\text { case 2: } u=u_{1}, w=u_{3}, r=u_{2}\end{array}$ & $\begin{array}{l}\text { case 3: } u=u_{2}, w=u_{1}, r=u_{3} \\
\text { case 4: } u=u_{2}, w=u_{3}, r=u_{1}\end{array}$ & $\begin{array}{l}v u_{1} \\
u_{2} \quad u_{3}\end{array}$ case $1: u=u_{3}, w=u_{1}, r=u_{2}$ \\
\hline & $\underbrace{v}_{u_{2}} u_{3}^{v}$ & $\begin{array}{l}\text { ase 1: } u=u_{1}, w=u_{2}, r=u_{3} \\
\text { ase 2: } u=u_{1}, w=u_{3}, r=u_{2} \\
\text { ase 3: } u=u_{2}, w=u_{1}, r=u_{3}\end{array}$ & $\begin{aligned} \text { case 4: } u=u_{2}, w=u_{3}, r=u_{1} \\
\text { case 5: } u=u_{3}, w=u_{1}, r=u_{2} \\
\text { case 6: } u=u_{3}, w=u_{2}, r=u_{1}\end{aligned}$ \\
\hline
\end{tabular}

Fig. 6 The ways of $\operatorname{Star} 3(v, G)$ sampling a CIS that includes $v$ in different orbits. Numbers in blue are orbit IDs. $u, w$, and $r$ in red are the variables in Algorithm 3. 


\subsection{Sampling Method $\operatorname{Path} 3(v, G)$}

Path3 $(v, G)$ first randomly samples a path of length 3 that includes $v$ as the second node, and then retrieves the 4-node subgraph's induced subgraph. Formally, Path3 $(v, G)$ consists of four steps:

Step 1. Sample a node $u$ from $N_{v}$ according to distribution $\boldsymbol{\alpha}^{(v)}$, which has the same definition as in method Path2 $(v, G)$. Here we do not sample $u$ from $N_{v}$ uniformly but according to $\boldsymbol{\alpha}^{(v)}$ to guarantee that the first three steps of Path3 $(v, G)$ randomly and uniformly samples a path of length 3 that includes $v$ as the second node, which facilitates estimation of the sampling bias;

Step 2. Sample a node $w$ from $N_{v}-\{u\}$ at random;

Step 3. Sample a node $r$ from $N_{u}-\{v\}$ at random;

Step 4. Return the CIS $s$ consisting of nodes $v, u, w$, and $r$.

Note that $s$ is a 3-node CIS when $w=r$. The pseudo-code of $\operatorname{Path} 3(v, G)$ is shown in Algorithm 2.

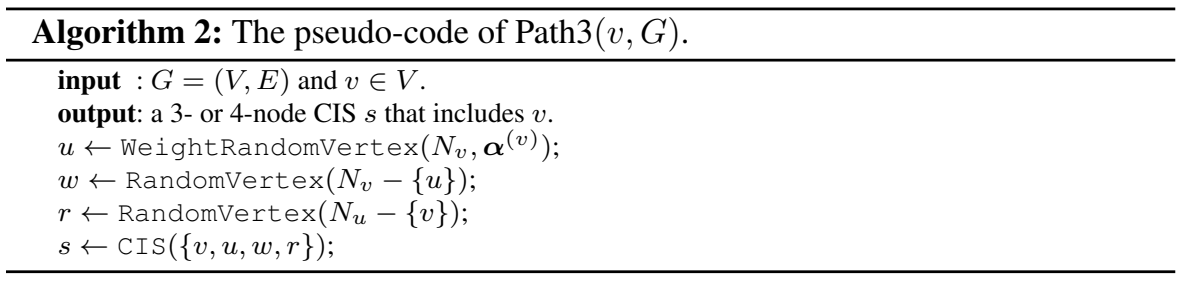

Theorem 5 Let $\check{\pi}_{i, v}$ denote the probability that method Path3 $(v, G)$ samples a 3-or 4-node CIS $s$ including $v$ in orbit $i \in\{1, \ldots, 14\}$. Denote $\check{\varphi}_{v}$ as the number of paths of length 3 including $v$ as the second node. Formally, $\check{\varphi}_{v}$ is computed as

$$
\check{\varphi}_{v}=\left(d_{v}-1\right) \varphi_{v} .
$$

Then $\check{\pi}_{1, v}=\check{\pi}_{2, v}=\check{\pi}_{4, v}=\check{\pi}_{6, v}=\check{\pi}_{7, v}=\check{\pi}_{9, v}=0, \check{\pi}_{3, v}=\frac{2}{\check{\varphi}_{v}}, \check{\pi}_{5, v}=\frac{1}{\check{\varphi}_{v}}$, $\check{\pi}_{8, v}=\frac{2}{\check{\varphi}_{v}}, \check{\pi}_{10, v}=\frac{1}{\check{\varphi}_{v}}, \check{\pi}_{11, v}=\frac{2}{\check{\varphi}_{v}}, \check{\pi}_{12, v}=\frac{2}{\check{\varphi}_{v}}, \check{\pi}_{13, v}=\frac{4}{\check{\varphi}_{v}}$, and $\pi_{14, v}=\frac{6}{\check{\varphi}_{v}}$.

Proof. The number of selections of variables $u, w$, and $r$ in Algorithm 2, i.e., the number of paths of length 3 that include $v$ as the second node, is $\left(d_{v}-1\right) \sum_{u \in N_{v}}\left(d_{u}-\right.$ $1)=\check{\varphi}_{v}$. As shown in Figure 5, Path $3(v, G)$ has $2,1,2,1,2,2,4$, and 6 ways to sample a 3 - or 4-node CIS $s$ including $v$ in orbits 3, 5, 8, 10, 11, 12, 13, and 14 respectively. Each way happens with probability $\alpha_{u}^{(v)} \times \frac{1}{d_{u}-1} \times \frac{1}{d_{v}-1}=\frac{1}{\check{\varphi}_{v}}$. When $s$ includes $v$ in the other orbits, Path $3(v, G)$ is not able to sample $s$. Therefore, we have $\check{\pi}_{1, v}=\check{\pi}_{2, v}=\check{\pi}_{4, v}=\check{\pi}_{6, v}=\check{\pi}_{7, v}=\check{\pi}_{9, v}=0, \check{\pi}_{3, v}=\frac{2}{\check{\varphi}_{v}}, \check{\pi}_{5, v}=\frac{1}{\check{\varphi}_{v}}, \check{\pi}_{8, v}=\frac{2}{\check{\varphi}_{v}}$, $\check{\pi}_{10, v}=\frac{1}{\check{\varphi}_{v}}, \check{\pi}_{11, v}=\frac{2}{\check{\varphi}_{v}}, \check{\pi}_{12, v}=\frac{2}{\check{\varphi}_{v}}, \check{\pi}_{13, v}=\frac{4}{\check{\varphi}_{v}}$, and $\pi_{14, v}=\frac{6}{\check{\varphi}_{v}}$. 


\subsection{Sampling Method $\operatorname{Star3}(v, G)$}

Denote $\tilde{\varphi}_{v}$ as the number of 4-node (not necessarily induced) subgraphs that are isomorphic to a 4-node star (i.e., graphlet $G_{4}$ ) and include $v$ as a leaf node. Formally, $\tilde{\varphi}_{v}$ is computed as

$$
\tilde{\varphi}_{v}=\sum_{u \in N_{v}}\left(\phi_{u}-d_{u}+1\right) .
$$

$\operatorname{Star} 3(v, G)$ first randomly samples a 4-node subgraph that is isomorphic to a 4-node star and includes $v$ as a leaf node, and then retrieves the 4-node subgraph's induced subgraph. Formally, $\operatorname{Star} 3(v, G)$ consists of four steps:

Step 1. Sample a node $u$ from $N_{v}$ according to distribution $\boldsymbol{\beta}^{(v)}=\left(\beta_{u}^{(v)}\right)_{u \in N_{v}}$, where we define

$$
\beta_{u}^{(v)}=\frac{\phi_{u}-d_{u}+1}{\tilde{\varphi}_{v}} ;
$$

Step 2. Sample a node $w$ from $N_{u}-\{v\}$ at random. Here we do not sample $u$ from $N_{v}$ uniformly but according to $\boldsymbol{\beta}^{(v)}$ to guarantee that the first three steps of $\operatorname{Star} 3(v, G)$ randomly and uniformly sample a 4-node subgraph that is isomorphic to a 4-node star and includes $v$ as a leaf node, which facilitates estimation of the sampling bias;

Step 3. Sample a node $r$ from $N_{u}-\{v, w\}$ at random;

Step 4. Return the CIS $s$ consisting of nodes $v, u, w$, and $r$.

The pseudo-code of our method $\operatorname{Star} 3(v, G)$ is shown in Algorithm 3, where function WeightRandom $\operatorname{Vertex}\left(N_{v}, \boldsymbol{\beta}^{(v)}\right)$ returns a node sampled from $N_{v}$ according to distribution $\boldsymbol{\beta}^{(v)}$, and function $\operatorname{Random} \operatorname{Vertex}\left(N_{u}-\{v, w\}\right)$ returns a node sampled from $N_{u}-\{v, w\}$ at random. In Appendix, we introduce the implementation of these two functions in detail. The following Theorem 6 states the sampling probability distribution of $\operatorname{Star} 3(v, G)$.

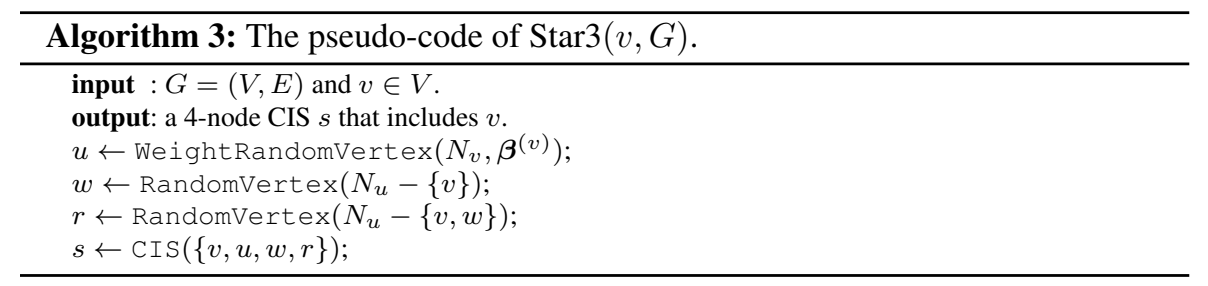

Theorem 6 Let $\tilde{\pi}_{i, v}$ denote the probability that method Star3 $(v, G)$ samples a 4node CIS $s$ including $v$ in orbit $i \in\{1, \ldots, 14\}$. Then $\tilde{\pi}_{1, v}=\tilde{\pi}_{2, v}=\tilde{\pi}_{3, v}=\tilde{\pi}_{4, v}=$ $\tilde{\pi}_{5, v}=\tilde{\pi}_{7, v}=\tilde{\pi}_{8, v}=\tilde{\pi}_{11, v}=0, \tilde{\pi}_{6, v}=\frac{1}{\tilde{\varphi}_{v}}, \tilde{\pi}_{9, v}=\frac{1}{\tilde{\varphi}_{v}}, \tilde{\pi}_{10, v}=\frac{1}{\tilde{\varphi}_{v}}, \tilde{\pi}_{12, v}=\frac{2}{\tilde{\varphi}_{v}}$, $\tilde{\pi}_{13, v}=\frac{1}{\tilde{\varphi}_{v}}$, and $\tilde{\pi}_{14, v}=\frac{3}{\tilde{\varphi}_{v}}$.

Proof. The number of selections of variables $u, w$, and $r$ in Algorithm 3 is $\sum_{u \in N_{v}}\left(d_{u}-\right.$ 1) $\left(d_{u}-2\right)=2 \tilde{\varphi}_{v}$. As shown in Figure 6 , $\operatorname{Star} 3(v, G)$ has 2, 2, 2, 4, 2, and 6 ways to sample a 4-node CIS $s$ including $v$ in orbits $6,9,10,12,13$, and 14 respectively. Each way happens with probability $\beta_{u}^{(v)} \times \frac{1}{d_{u}-1} \times \frac{1}{d_{u}-2}=\frac{1}{2 \tilde{\varphi}_{v}}$. When $s$ 
includes $v$ in the other orbits, $\operatorname{Star} 3(v, G)$ is not able to sample $s$. Therefore, we have $\tilde{\pi}_{1, v}=\tilde{\pi}_{2, v}=\tilde{\pi}_{3, v}=\tilde{\pi}_{4, v}=\tilde{\pi}_{5, v}=\tilde{\pi}_{7, v}=\tilde{\pi}_{8, v}=\tilde{\pi}_{11, v}=0, \tilde{\pi}_{6, v}=\frac{1}{\tilde{\varphi}_{v}}$, $\tilde{\pi}_{9, v}=\frac{1}{\tilde{\varphi}_{v}}, \tilde{\pi}_{10, v}=\frac{1}{\tilde{\varphi}_{v}}, \tilde{\pi}_{12, v}=\frac{2}{\tilde{\varphi}_{v}}, \tilde{\pi}_{13, v}=\frac{1}{\tilde{\varphi}_{v}}$, and $\tilde{\pi}_{14, v}=\frac{3}{\tilde{\varphi}_{v}}$.

\subsection{Orbit Degree Estimation}

For $i \in\{1, \ldots, 14\}$, let $m_{i}, \check{m}_{i}$, and $\tilde{m}_{i}$ denote the number of CISes sampled by $\operatorname{Path} 2(v, G)$, Path3 $(v, G)$, and $\operatorname{Star} 3(v, G)$ that include $v$ in orbit $i$ respectively. Then, we estimate $d_{v}^{(1)}, \ldots, d_{v}^{(14)}$ as:

Step 1. For orbit $i \in\{1,5,6,8,9,11\}$, according to Theorem 1 , we estimate $d_{v}^{(i)}$ as

$$
\hat{d}_{v}^{(i)}=\left\{\begin{array}{l}
\frac{m_{1}}{k \pi_{2, v}}, i=1, \\
\frac{\tilde{m}_{i}}{\tilde{k} \tilde{\pi}_{i, v}}, i \in\{5,8,11\}, \\
\frac{\tilde{m}_{i}}{\tilde{k} \tilde{\pi}_{i, v}}, i \in\{6,9\}
\end{array}\right.
$$

Step 2. For orbit 3, we compute two estimates $\check{d}_{v}^{(3)}=\frac{\check{m}_{3}}{\check{\pi}_{3, v}}$ and $\dot{d}_{v}^{(3)}=\frac{m_{3}}{k \pi_{3, v}}$. According to Theorem 1, these are unbiased estimates of $d_{v}^{(3)}$ and their variances are

$$
\begin{aligned}
& \operatorname{Var}\left(\check{d}_{v}^{(3)}\right)=\frac{d_{v}^{(3)}}{\check{k}}\left(\frac{1}{\check{\pi}_{3, v}}-d_{v}^{(3)}\right), \\
& \operatorname{Var}\left(\dot{d}_{v}^{(3)}\right)=\frac{d_{v}^{(3)}}{k}\left(\frac{1}{\pi_{3, v}}-d_{v}^{(3)}\right) .
\end{aligned}
$$

Theorem 2 allows us to compute a more accurate estimate

$$
\hat{d}_{v}^{(3)}=\lambda_{v}^{(3,1)} \check{d}_{v}^{(3)}+\lambda_{v}^{(3,2)} \dot{d}_{v}^{(3)},
$$

where $\lambda_{v}^{(3,1)}=\frac{\operatorname{Var}\left(\dot{d}_{v}^{(3)}\right)}{\operatorname{Var}\left(\check{d}_{v}^{(3)}\right)+\operatorname{Var}\left(\dot{d}_{v}^{(3)}\right)}$ and $\lambda_{v}^{(3,2)}=\frac{\operatorname{Var}\left(\breve{d}_{v}^{(3)}\right)}{\operatorname{Var}\left(\check{d}_{v}^{(3)}\right)+\operatorname{Var}\left(\dot{d}_{v}^{(3)}\right)}$ with $\operatorname{Var}\left(\check{d}_{v}^{(3)}\right)$ and $\operatorname{Var}\left(\tilde{d}_{v}^{(3)}\right)$ given by replacing $d_{v}^{(3)}$ with $\check{d}_{v}^{(3)}$ and $\tilde{d}_{v}^{(3)}$ in Eqs. (5) and (6);

Step 3. For orbit $i \in\{10,12,13,14\}$, we use Theorem 1 to compute two estimates $\check{d}_{v}^{(i)}=\frac{\check{m}_{i}}{\check{k}_{i, v}}$ and $\tilde{d}_{v}^{(i)}=\frac{\tilde{m}_{i}}{\tilde{k} \tilde{\pi}_{i, v}}$ with variances

$$
\begin{aligned}
& \operatorname{Var}\left(\check{d}_{v}^{(i)}\right)=\frac{d_{v}^{(i)}}{\check{k}}\left(\frac{1}{\check{\pi}_{i, v}}-d_{v}^{(i)}\right), \\
& \operatorname{Var}\left(\tilde{d}_{v}^{(i)}\right)=\frac{d_{v}^{(i)}}{\tilde{k}}\left(\frac{1}{\tilde{\pi}_{i, v}}-d_{v}^{(i)}\right) .
\end{aligned}
$$

We then apply Theorem 2 to compute a more accurate estimate

$$
\hat{d}_{v}^{(i)}=\lambda_{v}^{(i, 1)} \check{d}_{v}^{(i)}+\lambda_{v}^{(i, 2)} \tilde{d}_{v}^{(i)},
$$

where $\lambda_{v}^{(i, 1)}=\frac{\operatorname{Var}\left(\tilde{d}_{v}^{(i)}\right)}{\operatorname{Var}\left(\breve{d}_{v}^{(i)}\right)+\operatorname{Var}\left(\tilde{d}_{v}^{(i)}\right)}$ and $\lambda_{v}^{(i, 2)}=\frac{\operatorname{Var}\left(\breve{d}_{v}^{(i)}\right)}{\operatorname{Var}\left(\breve{d}_{v}^{(i)}\right)+\operatorname{Var}\left(\tilde{d}_{v}^{(i)}\right)}$ obtained by replacing $d_{v}^{(i)}$ with $\check{d}_{v}^{(i)}$ and $\tilde{d}_{v}^{(i)}$ in Eqs. (8) and (9); 
Step 4. For orbit $i \in\{2,4,7\}$, we now estimate $d_{v}^{(i)}$ as

$$
\begin{gathered}
\hat{d}_{v}^{(2)}=\phi_{v}-\hat{d}_{v}^{(3)}, \\
\hat{d}_{v}^{(4)}=\psi_{v}-2 \hat{d}_{v}^{(3)}-2 \hat{d}_{v}^{(8)}-2 \hat{d}_{v}^{(9)}-\hat{d}_{v}^{(10)}-4 \hat{d}_{v}^{(12)}-2 \hat{d}_{v}^{(13)}-6 \hat{d}_{v}^{(14)}, \\
\hat{d}_{v}^{(7)}=\gamma_{v}-\hat{d}_{v}^{(11)}-\hat{d}_{v}^{(13)}-\hat{d}_{v}^{(14)} .
\end{gathered}
$$

The following theorem presents the errors of the above estimates $\hat{d}_{v}^{(1)}, \ldots, \hat{d}_{v}^{(14)}$ for any node $v$ in graph $G$.

Theorem 7 For $i \in\{1, \ldots, 14\}, \hat{d}_{v}^{(i)}$ is an unbiased estimate of $d_{v}^{(i)}$ with the following variance.

I. For orbit $i \in\{1, \ldots, 14\}-\{2,4,7\}$, the variance of $\hat{d}_{v}^{(i)}$ is computed as

$$
\operatorname{Var}\left(\hat{d}_{v}^{(i)}\right)= \begin{cases}\frac{d_{v}^{(1)}}{k}\left(\frac{1}{\pi_{1, v}}-d_{v}^{(1)}\right), & i=1, \\ \frac{d_{v}^{(i)}}{\check{k}}\left(\frac{1}{\tilde{\pi}_{i, v}}-d_{v}^{(i)}\right), & i \in\{5,8,11\}, \\ \frac{d_{v}^{(i)}}{\tilde{k}}\left(\frac{1}{\tilde{\pi}_{i, v}}-d_{v}^{(i)}\right), & i \in\{6,9\}, \\ \frac{\operatorname{Var}\left(\tilde{d}_{v}^{(i)}\right) \operatorname{Var}\left(\check{d}_{v}^{(3)}\right)}{\operatorname{Var}\left(\check{(}_{v}^{(3)}\right)+\operatorname{Var}\left(\tilde{d}_{v}^{(3)}\right)}, & i=3, \\ \frac{\operatorname{Var}\left(\tilde{d}_{v}^{(i)}\right) \operatorname{Var}\left(\tilde{d}_{v}^{(i)}\right)}{\operatorname{Var}\left(\tilde{d}_{v}^{(i)}\right)+\operatorname{Var}\left(\tilde{d}_{v}^{(i)}\right)}, & i \in\{10,12,13,14\} .\end{cases}
$$

where $\operatorname{Var}\left(\tilde{d}_{v}^{(i)}\right), \operatorname{Var}\left(\tilde{d}_{v}^{(i)}\right)$, and $\operatorname{Var}\left(\check{d}_{v}^{(i)}\right)$ are defined in Eqs. (5), (6), (8), and (9). II. For orbit 2, the formula of $\operatorname{Var}\left(\hat{d}_{v}^{(2)}\right)$ equals that of $\operatorname{Var}\left(\hat{d}_{v}^{(3)}\right)$ derived above. III. For orbit 4, $\operatorname{Var}\left(\hat{d}_{v}^{(4)}\right)$ is computed as

$$
\begin{aligned}
& \operatorname{Var}\left(\hat{d}_{v}^{(4)}\right)=\sum_{j \in\{3,8,9,10,12,13,14\}} \chi_{j}^{2} \operatorname{Var}\left(\hat{d}_{v}^{(j)}\right) \\
& +\sum_{j, l \in\{3,8,9,10,12,13,14\} \wedge j \neq l} \chi_{j} \chi_{l} \operatorname{Cov}\left(\hat{d}_{v}^{(j)}, \hat{d}_{v}^{(l)}\right),
\end{aligned}
$$

where $\chi_{3}=\chi_{8}=\chi_{9}=\chi_{13}=2, \chi_{10}=1, \chi_{12}=4$, and $\chi_{14}=6$.

IV. For orbit 7, $\operatorname{Var}\left(\hat{d}_{v}^{(7)}\right)$ is computed as

$$
\begin{aligned}
\operatorname{Var}\left(\hat{d}_{v}^{(7)}\right) & =\operatorname{Var}\left(\hat{d}_{v}^{(11)}\right)+\operatorname{Var}\left(\hat{d}_{v}^{(13)}\right)+\operatorname{Var}\left(\hat{d}_{v}^{(14)}\right) \\
& +\sum_{j, l \in\{11,13,14\} \wedge j \neq l} \operatorname{Cov}\left(\hat{d}_{v}^{(j)}, \hat{d}_{v}^{(l)}\right) .
\end{aligned}
$$

The covariances in the formulas of $\operatorname{Var}\left(\hat{d}_{v}^{(4)}\right)$ and $\operatorname{Var}\left(\hat{d}_{v}^{(7)}\right)$ (i.e., Eqs. (11) and (12)) are computed as:

1. $\operatorname{Cov}\left(\hat{d}_{v}^{(3)}, \hat{d}_{v}^{(9)}\right)=\operatorname{Cov}\left(\hat{d}_{v}^{(8)}, \hat{d}_{v}^{(9)}\right)=0, \operatorname{Cov}\left(\hat{d}_{v}^{(3)}, \hat{d}_{v}^{(8)}\right)=-\frac{\lambda_{v}^{(3,1)} d_{v}^{(3)} d_{v}^{(8)}}{\hat{k}} ;$

2. When $j \in\{10,12,13,14\}$, we have $\operatorname{Cov}\left(\hat{d}_{v}^{(3)}, \hat{d}_{v}^{(j)}\right)=-\frac{\lambda_{v}^{(3,1)} \lambda_{v}^{(j, 1)} d_{v}^{(3)} d_{v}^{(j)}}{\check{k}}$ and $\operatorname{Cov}\left(\hat{d}_{v}^{(9)}, \hat{d}_{v}^{(j)}\right)=-\frac{\lambda_{v}^{(j, 2)} d_{v}^{(9)} d_{v}^{(j)}}{\tilde{k}}$;

3. When $j \in\{8,11\}$ and $l \in\{10,12,13,14\}, \operatorname{Cov}\left(\hat{d}_{v}^{(j)}, \hat{d}_{v}^{(l)}\right)=-\frac{\lambda_{v}^{(l, 1)} d_{v}^{(j)} d_{v}^{(l)}}{\dot{k}}$; 
4. When $j, l \in\{10,12,13,14\}$ and $j \neq l, \operatorname{Cov}\left(\hat{d}_{v}^{(j)}, \hat{d}_{v}^{(l)}\right)=-\frac{\lambda_{v}^{(j, 1)} \lambda_{v}^{(l, 1)} d_{v}^{(j)} d_{v}^{(l)}}{\dot{k}}-$ $\frac{\lambda_{v}^{(j, 2)} \lambda_{v}^{(l, 2)} d_{v}^{(j)} d_{v}^{(l)}}{\tilde{k}}$.

We give the proof of Theorem 7 in Appendix.

\section{Evaluation}

\subsection{Datasets}

We perform our experiments on publicly available graph datasets from the Stanford Network Analysis Platform (SNAP) ${ }^{2}$ : Flickr, Pokec, LiveJounal, YouTube, WikiTalk, and Web-Google, which are summarized in Table 4. We evaluate our method SNOD for computing the orbit degrees of nodes in the undirected graphs of datasets Flickr, Pokec, LiveJounal, YouTube, Wiki-Talk, and Web-Google, which are obtained by discarding edge directions.

Table 4 Graph datasets used in our experiments. "edges" refers to the number of edges in the undirected graph generated by discarding edge directions. "max-degree" represents the maximum number of edges incident to a node in the undirected graph.

\begin{tabular}{|c|ccc|}
\hline graph & nodes & edges & max-degree \\
\hline Flickr [24] & $1,715,255$ & $15,555,041$ & 27,236 \\
Pokec [34] & $1,632,803$ & $22,301,964$ & 14,854 \\
LiveJournal [24] & $5,189,809$ & $48,688,097$ & 15,017 \\
YouTube [24] & $1,138,499$ & $2,990,443$ & 28,754 \\
Wiki-Talk [20] & $2,394,385$ & $4,659,565$ & 100,029 \\
Web-Google [1] & 875,713 & $4,322,051$ & 6,332 \\
\hline
\end{tabular}

\subsection{Metric}

We use the normalized root mean square error (NRMSE) to measure the relative error of the orbit degree estimate $\hat{d}_{v}^{(i)}$ with respect to its true value $d_{v}^{(i)}, i=1,2, \ldots$ It is defined as $\operatorname{NRMSE}\left(\hat{d}_{v}^{(i)}\right)=\frac{\sqrt{\operatorname{MSE}\left(\hat{d}_{v}^{(i)}\right)}}{d_{v}^{(i)}}, i=1,2, \ldots$, where $\operatorname{MSE}\left(\hat{d}_{v}^{(i)}\right)$ denotes the mean square error of $\hat{d}_{v}^{(i)}: \operatorname{MSE}\left(\hat{d}_{v}^{(i)}\right)=\mathbb{E}\left(\left(\hat{d}_{v}^{(i)}-d_{v}^{(i)}\right)^{2}\right)=\operatorname{Var}\left(\hat{d}_{v}^{(i)}\right)+$ $\left(\mathbb{E}\left(\hat{d}_{v}^{(i)}\right)-d_{v}^{(i)}\right)^{2} \cdot \operatorname{MSE}\left(\hat{d}_{v}^{(i)}\right)$ is decomposed into a sum of the variance and bias of the estimator $\hat{d}_{v}^{(i)}$, both quantities are important and need to be as small as possible to achieve good estimation performance. When $\hat{d}_{v}^{(i)}$ is an unbiased estimator of $d_{v}^{(i)}$, we have $\operatorname{MSE}\left(\hat{d}_{v}^{(i)}\right)=\operatorname{Var}\left(\hat{d}_{v}^{(i)}\right)$. In our experiments, we average the estimates and calculate their NRMSEs over 1,000 runs. We evenly distribute the sampling budget among three sampling methods of SNOD, and leave the optimal budget distribution

2 www.snap.stanford.edu 
in future study. Our experiments are conducted on a server with a Quad-Core AMD Opeteron (tm) $8379 \mathrm{HE} \mathrm{CPU} 2.39 \mathrm{GHz}$ processor and $128 \mathrm{~GB}$ DRAM memory.

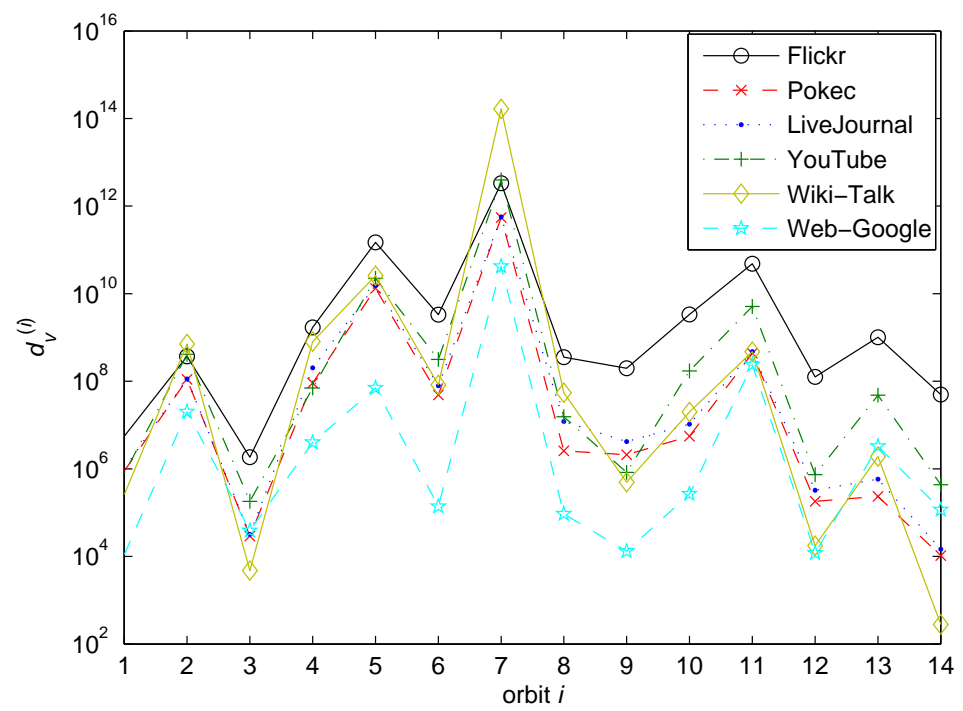

Fig. 7 Real values of 3- and 4-node orbit degrees of node $v_{\max }$.

\subsection{Results}

We evaluate the performance of SNOD for estimating 3- and 4-node orbit degrees over Flickr, Pokec, LiveJounal, and YouTube. Figure 7 shows the real values of 3- and 4-node orbit degrees of node $v_{\max }$ with the largest degree, where we use the state-ofthe-art enumeration method 4-Prof-Dist [11]. Roughly speaking, 3- and 4-node orbit degree distributions of graphs Flickr, Pokec, LiveJounal, and YouTube exhibit similar patterns. $d_{v_{\max }}^{(5)}, d_{v_{\max }}^{(7)}$, and $d_{v_{\max }}^{(11)}$ are the three largest 3- and 4-node orbit degrees.

Figure 8 shows the NRMSEs of estimates $\hat{d}_{v_{\max }}^{(i)}$ given by our method SNOD, $i=1, \ldots, 14$, where the sampling budget is $10^{6}$. Later in Table 6 , we will show that the computational cost is less than 1 minute to obtain these estimations for each of four graphs Flickr, Pokec, LiveJounal, and YouTube. We observe that all NRMSEs of $\hat{d}_{v_{\max }}^{(i)}$ are smaller than 0.1 for all these four graphs. The maximum of NRMSEs of all $\hat{d}_{v_{\max }}^{(i)}$, i.e., $\max _{i=1, \ldots, 14} \operatorname{NRMSE}\left(\hat{d}_{v_{\max }}^{(i)}\right)$, are $0.029,0.070,0.037$, and 0.040 for Flickr, Pokec, LiveJounal, and YouTube respectively. Figure 9 shows that the NRMSEs of SNOD decrease as the sampling budget increases. Roughly speaking, the NRMSEs decrease linearly with the square root of the sampling budget. Table 5 shows the results on all graphs in Table 4. 
As discussed in Section 4, we easily find that 1) degree of orbit 1 can only be estimated by Path2; 2) degrees of orbits 5, 8, and 11 can only be estimated by Path3; 3 ) degrees of orbits 6 and 9 can only be estimated by Star3;4) degrees of orbits 2 , 4 , and 7 can only be estimated by the joint of Path2, Path3, and Star3; 5) degree of orbit 3 can be estimated by both Path2 and Path3;6) degrees of orbits 10, 12, 13, and 14 can be estimated by both Path3 and Star3. Using the formulas of estimation variances derived in Section 4.4, Figure 10 compares the variances of Path2, Path3, and Star3 for estimating node $v_{\max }$ 's degrees at orbits $3,10,12,13$, and 14 under the same sampling budget. We can see that Path2 is orders of magnitude more accurate than Path3 for estimating degree of orbit 3, and Star3 is orders of magnitude more accurate than Path3 for estimating degrees of orbits 10, 12, 13, and 14. In summary, we find that: 1) Path2 is the best for estimating degrees of orbits 1 and 3;2) Path3 is the best for estimating degrees of orbits 5, 8, 10, 11, 12, 13, and 14; 3) Star3 is the best for estimating degrees of orbits 6 and 9; 4) estimating degrees of orbits 2, 4, and 7 needs samples given by the joint of methods Path2, Path3, and Star3. Moreover, we also apply SNOD with multi-cores for computing orbit degrees of top-100 nodes with the largest degrees. These high degree nodes are evenly distributed into different cores. From Figure 11, we can see that the run time almost deceases linearly as the number of cores increases.

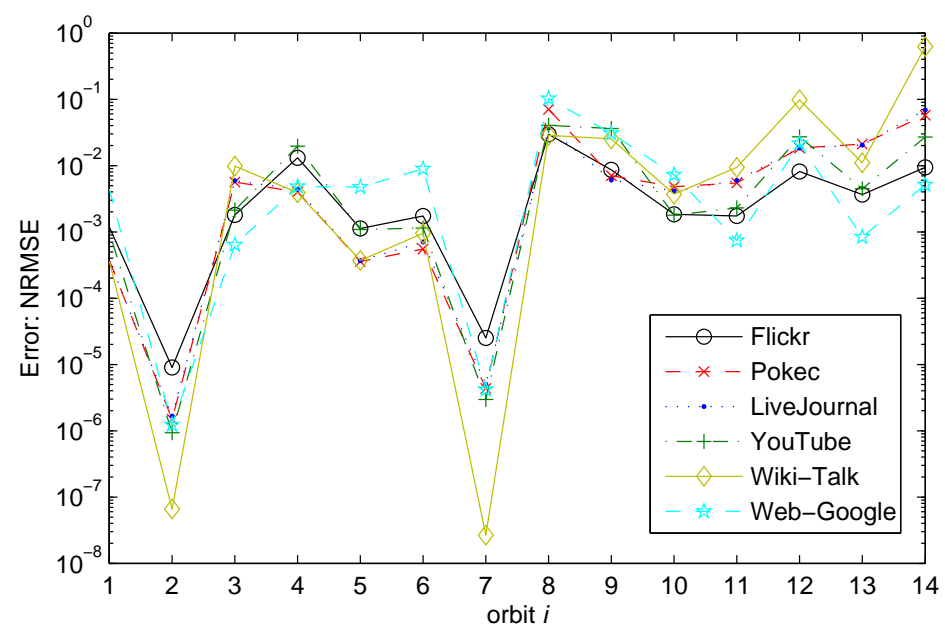

Fig. 8 NRMSEs of our estimates of 3- and 4-node orbit degrees of node $v_{\text {max }}$, where the sampling budget of SNOD is $10^{6}$

In addition, we also evaluate the performance of SNOD by comparing its performance to the state-of-the-art enumeration method 4-Prof-Dist [11] for estimating 3- and 4-node orbit degrees. Table 6 shows that our method SNOD takes 15 to 60 seconds to obtain accurate estimates of orbit degrees of the node $v_{\max }$, with NRMSEs less than 0.03 for all graphs. To exactly compute these orbit degrees, the state-of-theart method 4-Prof-Dist [11] requires 4 to 183 times more computational cost than 


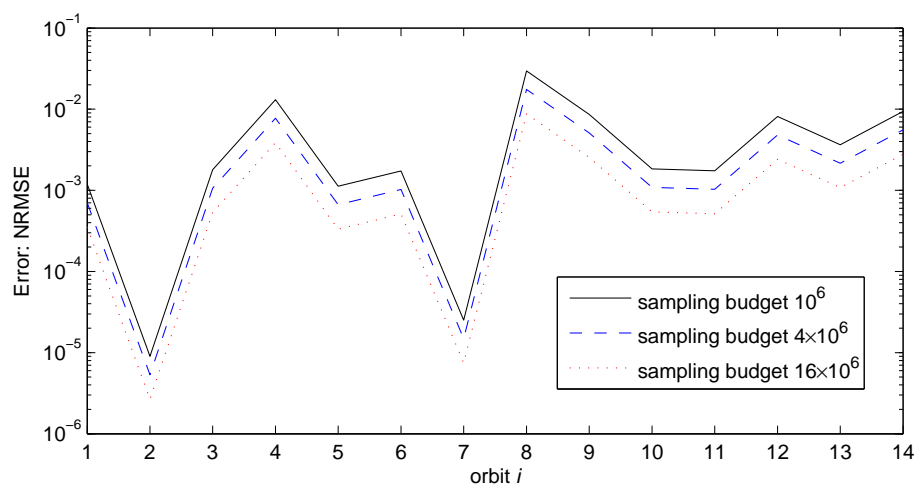

Fig. 9 (Flickr) NRMSEs of our estimates of 3- and 4-node orbit degrees of node $v_{\text {max }}$ for different sampling budget.

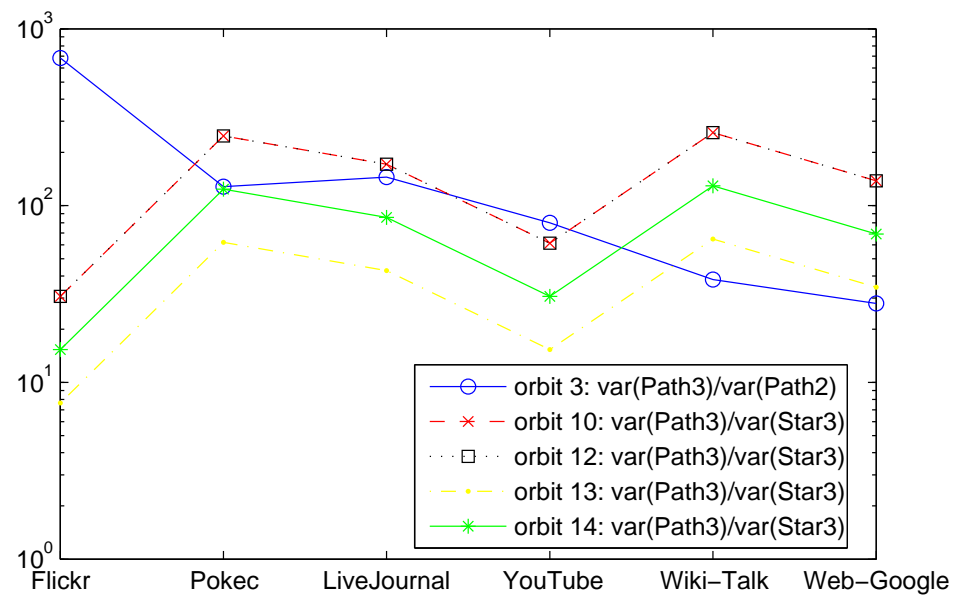

Fig. 10 Compared variances of Path2, Path3, and Star3 for estimating orbit degrees of node $v_{\max }$ under the same sampling budget.

our method. Table 7 shows that the results of our method SNOD for estimating all nodes' graphlet degrees in comparison with 4-Prof-Dist. For each node, we dynamically increase its sampled budget until the average of estimated variances is smaller than a desired threshold $\delta$. We can see that SNOD is 5 to 17 (resp. 2 to 6) faster than 4-Prof-Dist for $\delta=0.1$ (resp. $\delta=0.05$ ). In addition, we also apply state-of-the-art method wedge sampling [17] (resp. 3-path sampling [18]) to estimate global 3-node (resp. 4-node) graphlet counts with $\delta=0.1$ and $\delta=0.05$, and we find that SNOD is orders of magnitude slower than the joint of wedge sampling and 3-path sampling. It indicates that SNOD is not a suitable solution for the problem of global graphlet count approximation. 


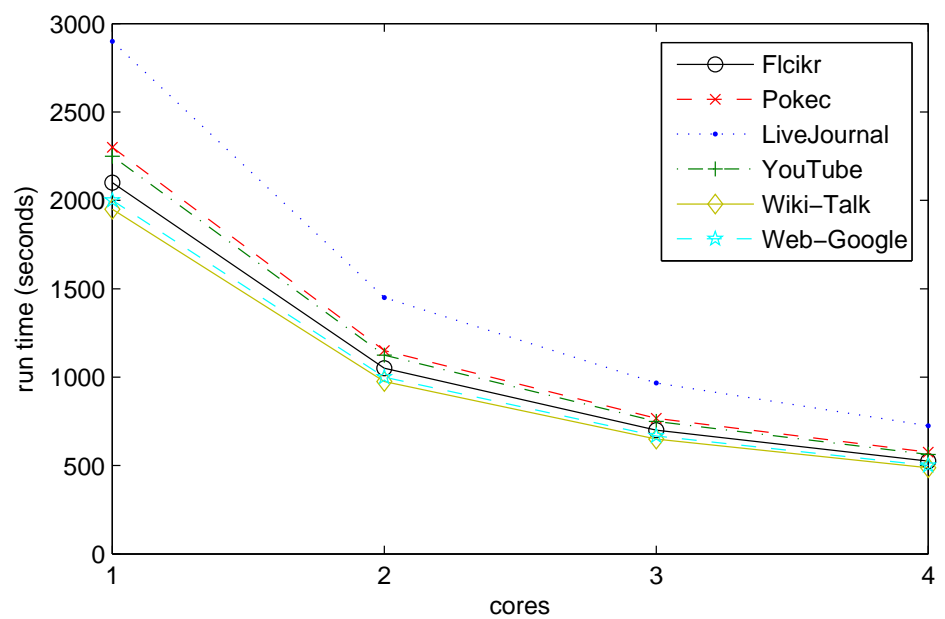

Fig. 11 The run time of SNOD with different number of cores.

Table 5 The maximum of NRMSEs of all 14 orbit degrees.

\begin{tabular}{|c|cc|}
\hline \multirow{2}{*}{ graph } & \multicolumn{2}{|c|}{ maximum NRMSE } \\
\cline { 2 - 3 } & sampling budget $=10^{6}$ & sampling budget $=4 \times 10^{6}$ \\
\hline Flickr & 0.029 & 0.015 \\
Pokec & 0.070 & 0036 \\
LiveJournal & 0.037 & 0.019 \\
YouTube & 0.040 & 0.021 \\
Wiki-Talk & 0.045 & 0.023 \\
Web-Google & 0.031 & 0.016 \\
\hline
\end{tabular}

Table 6 Computational cost and accuracy of SNOD for estimating orbit degrees of the node $v_{\max }$ in comparison with state-of-the-art method 4-Prof-Dist [11], where the sampling budget of SNOD is $10^{6}$.

\begin{tabular}{|c|c|c|c|}
\hline \multirow{2}{*}{ graph } & \multicolumn{2}{|c|}{ computational time (seconds) } & \multirow{2}{*}{$\begin{array}{l}\text { maximum NRMSE of SNOD, i.e., } \\
\max _{i=1, \ldots, 14} \operatorname{NRMSE}\left(\hat{d}_{v_{\max }}^{(i)}\right)\end{array}$} \\
\hline & 4-Prof-Dist [11] & SNOD & \\
\hline Flickr & 7,681 & 41.9 & 0.029 \\
\hline Pokec & 179 & 45.7 & 0.070 \\
\hline LiveJournal & 300 & 58.2 & 0.037 \\
\hline YouTube & 675 & 45.3 & 0.040 \\
\hline Wiki-Talk & 7,458 & 38.7 & 0.045 \\
\hline Web-Google & 91.5 & 40.1 & 0.031 \\
\hline
\end{tabular}

\section{Related Work}

Considerable attention has been paid to designing algorithms for exactly enumerating and counting triangles in large graphs $[8,33,31,10,11]$ and other high-order graphlets $[40,25,32]$. [21,10,11,3,27] reduce the computational time of counting 4- and 5-node graphlets by utilizing the relationships between 3-, 4-, and 5-node graphlet counts. To further accelerate the speed of counting graphlets, a number of 
Table 7 The number of times of speed up achieved by our method SNOD for estimating all nodes' graphlet orbit degrees in comparison with state-of-the-art method 4-Prof-Dist [11].

\begin{tabular}{|c|cc|}
\hline \multirow{2}{*}{ graph } & \multicolumn{2}{|c|}{ speed up } \\
\cline { 2 - 3 } & mean variance $\leq 0.1$ & mean variance $\leq 0.05$ \\
\hline Flickr & 15 & 3.7 \\
Pokec & 13 & 5.2 \\
LiveJournal & 12 & 6.1 \\
YouTube & 9.4 & 4.8 \\
Wiki-Talk & 17 & 3.1 \\
Web-Google & 5.3 & 2.4 \\
\hline
\end{tabular}

fast sampling methods have been developed for approximately computing a large graph's graphlet concentrations $[4,19,40,26,7,36]$ and graphlet counts $[4,35,29,5$, $16,2,18]$. To estimate graphlet concentrations, Kashtan et al. [19] propose a simple subgraph sampling method. However their method is computationally expensive when calculating the weight of each sampled subgraph, which is used for correcting bias introduced by edge sampling. To address this drawback, Wernicke [40] proposes a sampling method RAND-ESU based on randomly enumerating subgraph trees. These methods assume the entire topology of the graph of interest is known in advance and it can be fit into the memory. When the graph's topology is not available in advance and it is costly to sample the entire topology, Bhuiyan et al. [7] propose a Metropolis-Hastings based sampling method to estimate 3-, 4-, and 5-node graphlet concentrations ${ }^{3}$. Wang et al. [36] propose a more efficient crawling method to estimate online social network motif concentrations. When the available dataset is a set of random edges sampled from streaming graphs ${ }^{4}$, Wang et al. [37] propose an efficient crawling method to estimate graphlet concentrations.

The above sampling methods fail to compute graphlet counts, which is more fundamental than graphlet concentrations. Alon et al. [4] propose a color-coding method to reduce the computational cost of counting subgraphs. Color-coding reduces computation by coloring nodes randomly and enumerating only colorful CISes (i.e., CISes that consist of nodes with distinct colors), but [18] reveals that the colorcoding method is not scalable and is hindered by the sheer number of colorful CISes. Rahman et al. [29] develop a sampling method Graft to estimate 3-, 4-, and 5-node graphlet counts. Graft samples a fraction of edges uniformly. For a specific graphlet and each sampled edge, it computes a partial count for the graphlet with respect to the edge, i.e., the number of CISes that contain the edge and are isomorphic to the graphlet. Then, Graft uses sampled edges' partial graphlet counts to approximate the graphlet's total count. The drawback of Graft is its large computational cost in computing each sampled edge's partial graphlet count. $[35,5,16,2]$ develop fast sampling methods to estimate the number of triangles of static and dynamic graphs. Jha et al. [18] develop sampling methods to estimate counts of 4-node graphlets. We modify methods in [18] to meet the requirement of vertex-centric programming model,

\footnotetext{
3 The concentration of a particular $k$-node graphlet in a network refers to the ratio of the graphlet count to the total number of $k$-node CISes in the network, $k=3,4,5, \ldots$.

${ }^{4}$ Streaming graph is given in form of a stream of edges.
} 
which is required by most current graph computing systems, and develop a sampling method to estimate counts of 5-node motifs [38]. These methods are designed to sample all subgraphs, but not tailored to meet the need of sampling the subgraphs that include a given node. Elenberg et al. [11] develop a method to estimate counts of 4-node graphlets that include a given node based on random edge sampling, but their sampling method cannot be used to estimate node orbit degrees because the orbit of a node in a sampled CIS may be different from that of the node in the original CISes. We point out that method 4-Prof-Dist in [11] can be easily extended and used to compute the exact values of a node's 4-node orbit degrees. However, our experiments show that it requires several hours to compute a large degree node's obit degrees, therefore it is not scalable. To the best of our knowledge, we are the first to propose a sampling method for estimating a node's orbit degrees for large graphs. Recently, Rossi et al. [30] develop a method to exactly compute local 3-, 4-, and 5-node connected graphlet counts for an edge $(u, v)$, which are defined as the number of appearances of 3-, 4-, and 5-node connected graphlets that contain edge $(u, v)$. Note that a connected graphlet contains edge $(u, v)$ may not be a local graphlet, because nodes in the graphlet may not connect to $u$ or $v$. Besides connected graphlets, Dave et al. [9] further study to compute the non-connected graphlet counts. These two methods are designed for edge-centric graphlet statistics, which are different from vertex-centric graphlet orbit statistics studied in this paper. For example, there exist two different types of 3-node local connected graphlets for an edge, but three different orbits (orbits $1,2,3)$ that a node in 3 -node local connected graphlets may occupy.

\section{Conclusions and Future Work}

We develop computationally efficient sampling methods to estimate node orbit degrees for large graphs. We provide unbiased estimators of node orbit degrees, and derive simple and exact formulas for the variances of our estimators. Meanwhile, we conduct experiments on a variety of publicly available datasets, and experimental results show that our method accurately estimate the orbit degrees of nodes with large degrees in graphs with millions of edges within one minute. In future, we plan to extend SNOD to estimate 5-node (or even higher order) orbit degrees and investigate orbit degree signatures as features for various machine learning tasks.

\section{Acknowledgment}

The authors wish to thank the anonymous reviewers for their helpful feedback. The research presented in this paper is supported in part by National Key R\&D Program of China (2018YFC0830500), National Natural Science Foundation of China (U1301254, 61603290, 61602371), the Ministry of Education\&China Mobile Research Fund (MCM20160311), 111 International Collaboration Program of China, China Postdoctoral Science Foundation (2015M582663), Shenzhen Basic Research Grant (JCYJ20160229195940462, JCYJ20170816100819428), Natural Science Basic Research Plan in Shaanxi Province of China (2016JQ6034). 
Appendix

\section{Implementation Details}

We discuss our methods for implementing the functions in Algorithms 1, 2, and 3. We also analyze their computational complexities.

Initialization of $\phi_{v}, \varphi_{v}, \check{\varphi}_{v}, \tilde{\varphi}_{v}, \psi_{v}$, and $\gamma_{v}$ : For each node $v$, we store its degree $d_{v}$ and its neighbors' degrees in a list. Therefore, $O(1)$ and $O\left(d_{v}\right)$ operations are required to compute $\phi_{v}$ and $\varphi_{v}$ respectively. Similarly, one can easily find that $O\left(N_{v}\right)$, $O\left(N_{v}\right), O\left(\sum_{u \in N_{v}} d_{u}\right)$, and $O(1)$ operations are required to compute $\check{\varphi}_{v}, \tilde{\varphi}_{v}, \psi_{v}$, and $\gamma_{v}$ respectively.

$\operatorname{RandomVertex}\left(N_{v}-\{u\}\right)$ : We use an array $N_{v}\left[1, \ldots, d_{v}\right]$ to store the neighbors of $v$. Let $i_{v, u}$ denote the index of $u$ in the list $N_{v}\left[1, \ldots, d_{v}\right]$, i.e., $N_{v}\left[i_{v, u}\right]=u$. Then, function Random $\operatorname{Vertex}\left(N_{v}-\{u\}\right)$ includes the following steps:

- Step 1. Select a number $z$ from $\left\{1, \ldots, d_{v}\right\}-\left\{i_{v, u}\right\}$ at random;

- Step 2. Return $N_{v}[z]$.

Therefore, the computational complexity of RandomVertex $\left(N_{v}-\{u\}\right)$ is $O(1)$.

$\operatorname{Random} \operatorname{Vertex}\left(N_{v}-\{u, w\}\right)$ : Similarly, it includes the following steps:

- Step 1. Select a number $z$ from $\left\{1, \ldots, d_{v}\right\}-\left\{i_{v, u}, i_{v, w}\right\}$ at random;

- Step 2. Return $N_{v}[z]$.

Therefore, the computational complexity of RandomVertex $\left(N_{v}-\{u, w\}\right)$ is $O(1)$.

WeightRandom $\operatorname{Vertex}\left(N_{v}, \boldsymbol{\alpha}^{(v)}\right)$ : We store an array $A_{\alpha}^{(v)}$ in memory, where $A_{\alpha}^{(v)}[i]$ is defined as $A_{\alpha}^{(v)}[i]=\sum_{j=1}^{i}\left(d_{N_{v}[j]}-1\right), 1 \leq i \leq d_{v}$. Let $A_{\alpha}^{(v)}[0]=0$. Then, WeightRandom $\operatorname{Vertex}\left(N_{v}, \boldsymbol{\alpha}^{(v)}\right)$ includes the following steps:

- Step 1. Select a number $z$ from $\left\{1, \ldots, A_{\alpha}^{(v)}\left[d_{v}\right]\right\}$ at random;

- Step 2. Find $i$ such that

$$
A_{\alpha}^{(v)}[i-1]<z \leq A_{\alpha}^{(v)}[i]
$$

which is solved by binary search;

- Step 3. Return $N_{v}[i]$.

Therefore, the computational complexity of function WeightRandomVertex $\left(N_{v}, \boldsymbol{\alpha}^{(v)}\right)$ is $O\left(\log d_{v}\right)$.

WeightRandom $\operatorname{Vertex}\left(N_{v}, \boldsymbol{\beta}^{(v)}\right)$ : We store an array $A_{\beta}^{(v)}$ in memory, where $A_{\beta}^{(v)}[i]$ is defined as

$$
A_{\beta}^{(v)}[i]=\sum_{j=1}^{i}\left(\phi_{N_{v}[j]}-d_{N_{v}[j]}+1\right), \quad 1 \leq i \leq d_{v} .
$$

Let $A_{\beta}^{(v)}[0]=0$. Then, WeightRandomVertex $\left(N_{v}, \boldsymbol{\beta}^{(v)}\right)$ includes the following steps:

- Step 1. Select a number $z$ from $\left\{1, \ldots, A_{\beta}^{(v)}\left[d_{v}\right]\right\}$ at random; 
- Step 2. Find $i$ such that

$$
A_{\beta}^{(v)}[i-1]<z \leq A_{\beta}^{(v)}[i]
$$

which again is solved by binary search;

- Step 3. Return $N_{v}[i]$.

Therefore, the computational complexity of function WeightRandomVertex $\left(N_{v}, \boldsymbol{\beta}^{(v)}\right)$ is $O\left(\log d_{v}\right)$.

\section{Proof of Theorem 7}

According to Theorems 1 and 4, we have

$$
\operatorname{Var}\left(\hat{d}_{v}^{(1)}\right)=\frac{d_{v}^{(1)}}{k}\left(\frac{1}{\pi_{1, v}}-d_{v}^{(1)}\right) .
$$

According to Theorems 1 and 5, we have

$$
\operatorname{Var}\left(\hat{d}_{v}^{(i)}\right)=\frac{d_{v}^{(i)}}{\check{k}}\left(\frac{1}{\check{\pi}_{i, v}}-d_{v}^{(i)}\right), i \in\{5,8,11\} .
$$

According to Theorems 1 and 6, we have

$$
\operatorname{Var}\left(\hat{d}_{v}^{(i)}\right)=\frac{d_{v}^{(i)}}{\tilde{k}}\left(\frac{1}{\tilde{\pi}_{i, v}}-d_{v}^{(i)}\right), i \in\{6,9\} .
$$

By Theorem 2 and the definition of $\hat{d}_{v}^{(3)}, \hat{d}_{v}^{(10)}, \hat{d}_{v}^{(12)}, \hat{d}_{v}^{(13)}$, and $\hat{d}_{v}^{(14)}$ in Eqs. (7) and (10), we have

$$
\begin{aligned}
\operatorname{Var}\left(\hat{d}_{v}^{(i)}\right) & =\operatorname{Var}\left(\frac{\operatorname{Var}\left(\tilde{d}_{v}^{(i)}\right) \check{d}_{v}^{(i)}+\operatorname{Var}\left(\check{d}_{v}^{(i)}\right) \tilde{d}_{v}^{(i)}}{\operatorname{Var}\left(\check{d}_{v}^{(i)}\right)+\operatorname{Var}\left(\tilde{d}_{v}^{(i)}\right)}\right) \\
& =\frac{\operatorname{Var}\left(\tilde{d}_{v}^{(i)}\right) \operatorname{Var}\left(\check{d}_{v}^{(i)}\right)}{\operatorname{Var}\left(\check{d}_{v}^{(i)}\right)+\operatorname{Var}\left(\tilde{d}_{v}^{(i)}\right)}, \quad i \in\{3,10,12,13,14\}
\end{aligned}
$$

In the above derivation, the last equation holds because $\breve{d}_{v}^{(i)}$ and $\tilde{d}_{v}^{(i)}$ are independent, which can be easily obtained from their definition in Eqs. (5), (6), (8), and (9). For $\operatorname{Var}\left(\hat{d}_{v}^{(2)}\right)$, we have

$$
\operatorname{Var}\left(\hat{d}_{v}^{(2)}\right)=\operatorname{Var}\left(\phi_{v}-\hat{d}_{v}^{(3)}\right)=\operatorname{Var}\left(\hat{d}_{v}^{(3)}\right) .
$$

By the definition of $\hat{d}_{v}^{(4)}$ and $\hat{d}_{v}^{(7)}$, we easily proof that their variances are

$$
\begin{aligned}
\operatorname{Var}\left(\hat{d}_{v}^{(4)}\right) & =\sum_{j \in\{3,8,9,10,12,13,14\}} \chi_{j}^{2} \operatorname{Var}\left(\hat{d}_{v}^{(j)}\right) \\
& +\sum_{j, l \in\{3,8,9,10,12,13,14\} \wedge j \neq l} \chi_{j} \chi_{l} \operatorname{Cov}\left(\hat{d}_{v}^{(j)}, \hat{d}_{v}^{(l)}\right),
\end{aligned}
$$




$$
\begin{aligned}
\operatorname{Var}\left(\hat{d}_{v}^{(7)}\right) & =\operatorname{Var}\left(\hat{d}_{v}^{(11)}\right)+\operatorname{Var}\left(\hat{d}_{v}^{(13)}\right)+\operatorname{Var}\left(\hat{d}_{v}^{(14)}\right) \\
& +\sum_{j, l \in\{11,13,14\} \wedge j \neq l} \operatorname{Cov}\left(\hat{d}_{v}^{(j)}, \hat{d}_{v}^{(l)}\right)+\sum_{j, l \in\{11,13,14\} \wedge j \neq l} \operatorname{Cov}\left(\hat{d}_{v}^{(j)}, \hat{d}_{v}^{(l)}\right) .
\end{aligned}
$$

The covariances in the above formulas of $\operatorname{Var}\left(\hat{d}_{v}^{(4)}\right)$ and $\operatorname{Var}\left(\hat{d}_{v}^{(7)}\right)$ are computed based on the following properties: (1) the covariance of estimates given by different sampling methods (e.g., Path2, Path3, and Star3) is zero; (2) the covariance of estimates given by the same sampling method is computed based on Theorem 1. For example, when $j, l \in\{10,12,13,14\}$ and $j \neq l$, by the definition of $\hat{d}_{v}^{(j)}$ in Eq. (10), we have $\operatorname{Cov}\left(\hat{d}_{v}^{(j)}, \hat{d}_{v}^{(l)}\right)=\operatorname{Cov}\left(\lambda_{v}^{(j, 1)} \breve{d}_{v}^{(j)}+\lambda_{v}^{(j, 2)} \tilde{d}_{v}^{(j)}, \lambda_{v}^{(l, 1)} \breve{d}_{v}^{(l)}+\lambda_{v}^{(l, 2)} \tilde{d}_{v}^{(l)}\right)$. By the definition of $\breve{d}_{v}^{(j)}$ and $\tilde{d}_{v}^{(j)}$ in Eqs. (8) and (9), we easily find that $\breve{d}_{v}^{(j)}$ and $\breve{d}_{v}^{(l)}$ given by sampling method Path3 are independent with $\tilde{d}_{v}^{(j)}$ and $\tilde{d}_{v}^{(l)}$ given by sampling method Star3. Moreover, we have $\operatorname{Cov}\left(\check{d}_{v}^{(j)}, \breve{d}_{v}^{(l)}\right)=-\frac{d_{v}^{(j)} d_{v}^{(l)}}{\dot{k}}$ and $\operatorname{Cov}\left(\tilde{d}_{v}^{(j)}, \tilde{d}_{v}^{(l)}\right)=-\frac{d_{v}^{(j)} d_{v}^{(l)}}{\tilde{k}}$ from Theorem 1. Thus, we have $\operatorname{Cov}\left(\hat{d}_{v}^{(j)}, \hat{d}_{v}^{(l)}\right)=-\frac{\lambda_{v}^{(j, 1)} \lambda_{v}^{(l, 1)} d_{v}^{(j)} d_{v}^{(l)}}{\dot{k}}-\frac{\lambda_{v}^{(j, 2)} \lambda_{v}^{(l, 2)} d_{v}^{(j)} d_{v}^{(l)}}{\tilde{k}}$.

\section{References}

1. Google programming contest. http://www.google.com/programming-contest/(2002)

2. Ahmed, N., Duffield, N., Neville, J., Kompella, R.: Graph sample and hold: A framework for biggraph analytics. In: KDD, pp. 589-597 (2014)

3. Ahmed, N.K., Neville, J., Rossi, R.A., Duffield, N.: Efficient graphlet counting for large networks. In: ICDM (2015)

4. Alon, N., Yuster, R., Zwick, U.: Color-coding. J. ACM 42(4), 844-856 (1995). DOI 10.1145/210332. 210337. URL http: //doi.acm.org/10.1145/210332.210337

5. A.Pavany, Tangwongsan, K., Tirthapuraz, S., Wu, K.L.: Counting and sampling triangles from a graph stream. In: PVLDB, pp. 1870-1881 (2013)

6. Benson, A.R., Gleich, D.F., Leskovec, J.: Higher-order organization of complex networks. Science 353(6295), 163-166 (2016)

7. Bhuiyan, M.A., Rahman, M., Rahman, M., Hasan, M.A.: Guise: Uniform sampling of graphlets for large graph analysis. In: ICDM, pp. 91-100 (2012)

8. Chu, S., Cheng, J.: Triangle listing in massive networks and its applications. In: KDD, pp. 672-680 (2011)

9. Dave, V.S., Ahmed, N.K., Hasan M.H.: E-CLoG: Counting edge-centric local graphlets. In: BigData, pp. 586-595 (2017)

10. Elenberg, E.R., Shanmugam, K., Borokhovich, M., Dimakis, A.G.: Beyond triangles: A distributed framework for estimating 3-profiles of large graphs. In: KDD, pp. 229-238 (2015)

11. Elenberg, E.R., Shanmugam, K., Borokhovich, M., Dimakis, A.G.: Distributed estimation of graph 4-profiles. In: WWW (2016)

12. Fang, M., Yin, J., Zhu, X., Zhang, C.: Trgraph: Cross-network transfer learning via common signature subgraphs. TKDE 27(9), 2536-2549 (2015)

13. Graybill, F.A., Deal, R.B.: Combining unbiased estimators. Biometrics 15(4), 543-550 (1959)

14. Grover, A., Leskovec, J.: node2vec: Scalable feature learning for networks. In: KDD (2016)

15. Henderson, K., Gallagher, B., Eliassi-Rad, T., Tong, H., Basu, S., Akoglu, L., Koutra, D., Faloutsos, C., Li, L.: Rolx: Structural role extraction and mining in large graphs. In: KDD, pp. 1231-1239 (2012)

16. Jha, M., Seshadhri, C., Pinar, A.: A space efficient streaming algorithm for triangle counting using the birthday paradox. In: KDD, pp. 589-597 (2013)

17. Seshadhri, C., Pinar, A., Kolda, T.G.: Wedge sampling for computing clustering coefficients and triangle counts on large graphs. Statistical Analysis and Data Mining, 7(4), 294-307 (2014) 
18. Jha, M., Seshadhri, C., Pinar, A.: Path sampling: A fast and provable method for estimating 4-vertex subgraph counts. In: WWW, pp. 495-505 (2015)

19. Kashtan, N., Itzkovitz, S., Milo, R., Alon, U.: Efficient sampling algorithm for estimating subgraph concentrations and detecting network motifs. Bioinformatics 20(11), 1746-1758 (2004)

20. Leskovec, J., Huttenlocher, D., Kleinberg, J.: Predicting positive and negative links in online social networks. In: WWW, pp. 641-650 (2010)

21. Marcus, D., Shavitt, Y.: Rage-A rapid graphlet enumerator for large networks. Computer Networks 56(2), 810-819 (2012)

22. Milenkovic, T., Memisevic, V., Ganesan, A.K., Przulj, N.: Systems-level cancer gene identification from protein interaction network topology applied to melanogenesis-related functional genomics data. Journal of The Royal Society Interface 7(44), 423-437 (2010)

23. Milenkovic, T., Przulj, N.: Uncovering biological network function via graphlet degree signatures. Cancer Informatics 6, 257-273 (2008)

24. Mislove, A., Marcon, M., Gummadi, K.P., Druschel, P., Bhattacharjee, B.: Measurement and analysis of online social networks. In: IMC, pp. 29-42 (2007)

25. Oliveira Aparicio, D., Pinto Ribeiro, P.M., Silva, F.M.A.D.: Parallel subgraph counting for multicore architectures. In: ISPA, pp. 34-41 (2014)

26. Omidi, S., Schreiber, F., Masoudi-nejad, A.: Moda: An efficient algorithm for network motif discovery in biological networks. Genes and Genet systems 84(5), 385-395 (2009)

27. Pinar, A., Seshadhr, C., Visha, V.: Escape: Efficiently counting all 5-vertex subgraphs. In: WWW (2017)

28. Przulj, N.: Biological network comparison using graphlet degree distribution. Bioinformatics 23(2), pp. 177-183 (2007)

29. Rahman, M., Bhuiyan, M., Hasan, M.A.: Graft: An approximate graphlet counting algorithm for large graph analysis. In: CIKM (2012)

30. Rossi, R.A., Zhou R., Ahmed, N.K.: Estimation of Graphlet Statistics. CoRR abs/1701.01772 (2017)

31. Schank, T.: Algorithmic aspects of triangle-based network analysis. Phd in Computer Science (2007)

32. Shao, Y., Cui, B., Chen, L., Ma, L., Yao, J., Xu, N.: Parallel subgraph listing in a large-scale graph. In: SIGMOD, pp. 625-636 (2014)

33. Suri, S., Vassilvitskii, S.: Counting triangles and the curse of the last reducer. In: WWW, pp. 607-614 (2011)

34. Takac, L., Zabovsky, M.: Data analysis in public social networks. In: International Scientific Conference and International Workshop Present Day Trends of Innovations, pp. 1-6 (2012)

35. Tsourakakis, C.E., Kang, U., Miller, G.L., Faloutsos, C.: Doulion: Counting triangles in massive graphs with a coin. In: KDD (2009)

36. Wang, P., Lui, J.C., Zhao, J., Ribeiro, B., Towsley, D., Guan, X.: Efficiently estimating motif statistics of large networks. TKDD 9(2), pp. 8:1-8:27 (2014)

37. Wang, P., Lui, J.C.S., Towsley, D.: Minfer: Inferring motif statistics from sampled edges. In: ICDE (2015)

38. Wang, P., Tao, J., Zhao, J., Guan, X.: Moss: A scalable tool for efficiently sampling and counting 4and 5-node graphlets. CoRR abs/1509.08089 (2015). URL http://arxiv. org/abs/1509. 08089

39. Wei, B., Liu, J., Ma, J., Zheng, Q., Zhang, W., Feng, B.: Motif-based hyponym relation extraction from wikipedia hyperlinks. TKDE 26(10), 2507-2519 (2014)

40. Wernicke, S.: Efficient detection of network motifs. IEEE/ACM Transactions on Computational Biology and Bioinformatics 3(4), 347-359 (2006)

41. Ye, J., Cheng, H., Zhu, Z., Chen, M.: Predicting positive and negative links in signed social networks by transfer learning. In: WWW, pp. 1477-1488 (2013) 

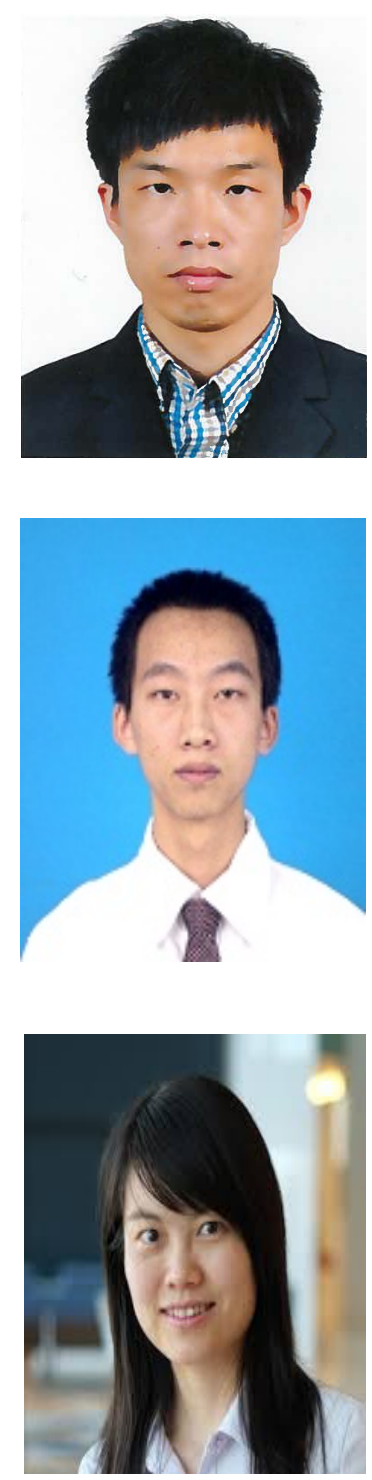

Pinghui Wang received the B.S. degree in information engineering and the Ph.D. degree in automatic control from Xi'an Jiaotong University, Xi' an, China, in 2006 and 2012 respectively. He is currently an associate professor in MOE Key Laboratory for Intelligent Networks and Network Security at Xi'an Jiaotong University. His research interests include Internet traffic measurement and modeling, abnormal detection, and online social network measurement.

Junzhou Zhao received the B.S. degree in information engineering and the Ph.D. degree in automatic control from Xi' an Jiaotong University, Xi' an, China, in 2008 and 2015 respectively. He is currently a Postdoc Fellow in the Division of Computer, Electrical and Mathematical Sciences and Engineering, King Abdullah University of Science and Technology, Saudi Arabia. His research interests include online social network measurement and modeling.

Xiangliang Zhang received the Ph.D. degree in computer science from INRIA-Universite Paris-Sud, France in 2010. She is currently an associate professor and directs the Machine Intelligence and knowledge Engineering (MINE) Laboratory in King Abdullah University of Science and Technology (KAUST), Saudi Arabia. Her main research interests and experiences are in machine learning, data mining, and cloud computing. 


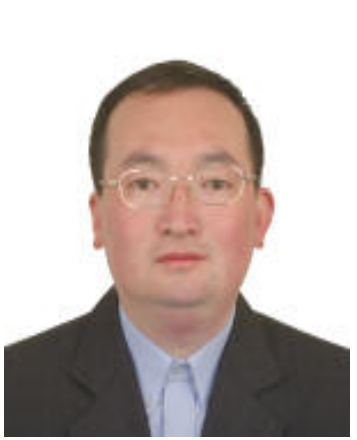

Jing Tao received the B.S and M.S degrees in automatic control from Xi' an Jiaotong University, Xi' an, China, in 2001 and 2006 respectively. $\mathrm{He}$ is currently a teacher in Xi' an Jiaotong University and on-the-job $\mathrm{Ph} . \mathrm{D}$. candidate with the Systems Engineering Institute and SKLMS Laboratory, Xi' an Jiaotong University under the supervision of Prof. Xiaohong Guan. His research interests include Internet traffic measurement and modeling, traffic classification, abnormal detection, and botnet.

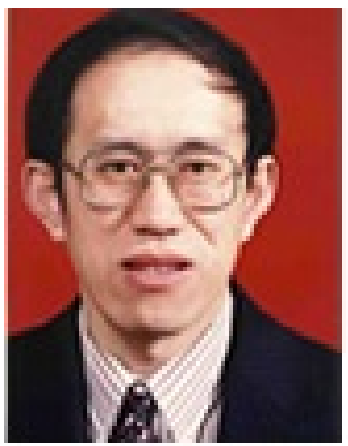

Xiaohong Guan received the B.S. and M.S. degrees in automatic control from Tsinghua University, Beijing, China, in 1982 and 1985, respectively, and the Ph.D. degree in electrical engineering from the University of Connecticut, Storrs, US, in 1993. He is currently a professor at the Systems Engineering Institute, Xi' an Jiaotong University, Xi' an, China. His research interests include allocation and scheduling of complex networked resources, network security, and sensor networks. 